\title{
Inactivación de la Dihidrolipoamida Deshidrogenasa de Trypanosoma cruzi por Fenotiazinas en presencia de Citocromo c y Peróxido de Hidrógeno. Efectos de losAntioxidantes
}

\author{
JOSÉ GUTIÉRREZ-CORREA*, y ANDRÉS O.M. STOPPANI**
}

\begin{abstract}
Trypanosoma cruzi DIHYDROLIPOAMIDE DEHYDROGENASE IS INACTIVATED BY PHENOTHIAZINES IN THE PRESENCE OF CYTOCHROME C AND HYDROGEN PEROXIDE. EFFECTS OF ANTIOXIDANTS
\end{abstract}

\begin{abstract}
Cytochrome c catalyzed the oxidation of phenothiazines (PTZ) in the presence of hydrogen peroxide. The transient formation of the promazine radical cation $\left(P Z^{+}\right)$has been demonstrated by light absorption measurements as well as by its conversión to promazine sulfoxide. Trypanosoma cruzi dihydrolipoamide dehydrogenase ( $L A D H T c$ ) was irreversibly inhibited by treatment with cytochrome c (cyt c)/ $\mathrm{H}_{2} \mathrm{O}_{2}$ system supplemented with PTZ. LADH T c inactivation depended on a) The PTZ structure b) Time of incubación with the complete oxidant system c) The presence of an antioxidant that intercept free radicals. PZ, thioridazine (TRDZ) and trimeprazine (TMPZ), were the most effective systems out of twelve PTZ studied, with inactivation values of 82,76 and $72 \%$, respectively, after 90 min of incubation. LADH T c inactivation by $P Z$ (with alkylamine substituent at $N 10$ position) decreased by its structural modification at 2 position (inactivation $P Z>$ chlorpromazine $(C P Z)>$ propionylpromazine (PPZ)>trifluopromazine (TFPZ)) or at $N 10$ position (inactivation $P Z>T M P Z>$ promethazine (PMTZ)) PTZ activity with piperidinyl substituent at N10 position depended on the group at 2 position (TRDZ, with thiomethyl group, has high inactivating effect on LADH T c; propericyazine (PCYZ), with cyano group, is much less active). Apparently, piperazinyl substituent at the N10 position on the phenothiazine have not an important function in the compound's inactivating effect on LADH T c. The effect of $P T Z$ with $\mathrm{Cl}$ at 2 position (CPZ, prochlorperazine (PCP), perphenazine (PFZ)) was higher than the effect of compounds with $\mathrm{CF}_{3}$ in the same position (TFPZ,trifluoperazine (TFP), fluphenazine (FFZ) ) independent on the structure of substituents at N10 position. Production of PTZ ${ }^{+}$radicals was essential for $L A D H T$ c inactivation and this effect depended on the stability of these free radicals. Comparision of inactivation values for LADH T $c$ and mammalian LADH demonstrated a greater sensitivity of LADH T c to various PTZ studied. Thiol compounds (such as GSH and N-acetylcysteine), tyrosine, tryptophan, NADH, ascorbate and trolox prevented LADH Tc
\end{abstract}

Instituto de Medicina Tropical Daniel A. Carrión. Facultad de Medicina. Universidad Nacional Mayor de San Marcos. Lima, Perú.

** Centro de Investigaciones Bioenergéticas. Facultad de Medicina (UBA-CONICET) Buenos Aires, Argentina. Correspondencia: Instituto de Medicina Tropical. Ciudad Universitaria (UNMSM). Av. Colonial Cdra 53. LimaPerú. Fax (14) 51032.E-mail: josegutierrezc@speedy.com.pe 
inactivation by the cyt $\mathrm{c} / \mathrm{H}_{2} \mathrm{O}_{2} / \mathrm{PTZ}$ systems in agreement with their ability for to suppress $\mathrm{PTZ}^{+}$ radicals. The role of $\mathrm{PTZ}^{+.}$as enzyme inhibitors, or as generators of secondary free radicals and metabolite depletors for phenothiazines cytotoxicity is discussed.

Key words: Phenothiazines, Trypanosoma cruzi, Dihydrolipoamide Dehydrogenase, Cytochrome c.

\section{INTRODUCCIÓN}

La enfermedad de Chagas es una infección parasitaria crónica, endémica en extensas áreas de América-entre el norte de Méjico y el sur de Argentina-que tiene como agente etiológico al Trypanosoma cruzi $(T c)$. Recientemente se ha estimado que 18-20 millones de personas están infectadas y que anualmente se producen aproximadamente 200.000 nuevos $\operatorname{casos}^{1,2}$. El tratamiento actual de esta enfermedad no es totalmente efectivo y suele producir severos efectos adversos. Estas limitaciones justifican la búsqueda de nuevos agentes terapéuticos, o la reevaluación de fármacos de efectos tripanocidas, orientados a una quimioterapia selectiva de esta enfermedad $^{1,3}$. Las fenotiazinas (FTZ) tienen múltiples actividades biológicas, destacando los efectos antipsicóticos, antibacterianos, antiparasitarios, antiretrovirales y antimultidrogas resistencia ${ }^{4,5}$. Entre los efectos antiparasitarios de FTZ se encuentran los relacionados al $T c$, como el aumento del AMP cíclico ${ }^{6}$, la inhibición de la cAMP fosfodiesterasa ${ }^{7}$, los cambios morfológicos de la membrana celular ${ }^{8}$, la desorganización mitocondrial $^{9,10}$, la modificación de la evolución de la enfermedad de Chagas experimental ${ }^{11}$, la inactivación de la tripanotiona reductasa $(\mathrm{TR})^{12,13}$ y de la dihidrolipoamida deshidrogenasa $(\mathrm{LADH})^{14}$. En trabajos previos demostramos que la mieloperoxidasa (MPO) y la mioglobina (Mb) modificada por el peróxido de hidrógeno, catalizaron la producción de radicales libres catiónicos de FTZ $\left(\mathrm{FTZ}^{+}\right)$, los cuales inhibieron irreversiblemente la TR y LADH del $T c^{13,14}$. Estos resultados sugieren que los radicales $\mathrm{FTZ}^{+}$. pueden contribuir a la acción tripanocida de FTZ. En el presente estudio hemos extendido nuestras observaciones al efecto del citocromo c(cit c), en presencia de $\mathrm{H}_{2} \mathrm{O}_{2}$ y FTZ, sobre la actividad LADH de $T c$. Esta enzima, componente esencial de los complejos multienzimáticos piruvato, $\alpha$ ceto glutarato y $\alpha$ ceto ácidos de cadena ramificada deshidrogenasas, posee dos centros catalíticos: el FAD y el disulfuro redox, y cataliza la reducción reversible de la lipoamida, L( S ) por el NADH, (Reacción1), a dihidrolipoamida, $\mathrm{L}(\mathrm{SH})_{2}{ }^{15}$.

$\mathrm{NADH}+\mathrm{H}^{+}+\mathrm{L}(\mathrm{S})_{2} \leftrightarrow \mathrm{NAD}^{+}+\mathrm{L}(\mathrm{SH})_{2}$

Estos complejos multienzimáticos tienen un rol principal en el metabolismo oxidativo del $T$ $c^{16}$. La LADH se encuentra en todos los estadíos de desarrollo del T. cruzi $^{17}$, en concordancia con la actividad del ciclo del ácido cítrico durante todo el ciclo evolutivo del parásito.

El cit c es una pequeña hemoproteína intermembranas que, formando parte de la cadena respiratoria mitocondrial, transporta electrones entre los complejos III (ubiquinol-cit c reductasa) y IV (citocromo oxidasa). El grupo prostético hemo del cit c está unido covalentemente a la cadena polipeptídica, muy básica, por dos enlaces tioéter que involucran a la $\mathrm{Cys}^{14}$ y $\mathrm{Cys}^{17}$. El solvente es muy poco accesible al grupo hemo, el cual se encuentra cerca del extremo N-terminal en un ambiente hidrofóbico, formando con la $\operatorname{His}^{18}$ y la $\operatorname{Met}^{80}$ el $5^{\circ}$ y $6^{\circ}$ ligandos, respectivamente, del $\mathrm{Fe}^{18-20}$. El cit c además de su función en el metabolismo energético participa, al ser liberado desde las mitocondrias al citosol, en el control de la apoptosis, causando interrupción del flujo de electrones en la cadena respiratoria, aumento de la generación de superóxido, la formación del apoptosoma, la activación de las caspasas y la muerte celular ${ }^{21,22}$. El cit c reacciona con hidroperóxidos ${ }^{23-26}$ y puede inducir la peroxidación de los lípidos y la modificación de proteínas ${ }^{27}$. Asi mismo, el cit c puede catalizar en presencia de peróxido de hidrógeno, la oxidación de diversas moléculas como el 2-ceto-4-tiometil butírico ${ }^{28}$, linoleato ${ }^{29}$, 2-2'-azino-bis(3-etilbenzotiazolino-6 ácido sulfónico (ABTS), 4-amino antipirina y luminol ${ }^{30}$, $\mathrm{N}$-metilcarbazol y tioanisol ${ }^{31}$, diclorofluorescina $^{32,33}$, benceno ${ }^{34}$, y otras incluyendo catecolaminas $^{35}$. El cit c se encuentra en altas concentraciones en el espacio íntermembranas mitocondriales, entre $0,5-5,0 \mathrm{mM}^{36} \mathrm{y}$ aunque 
normalmente la concentración de $\mathrm{H}_{2} \mathrm{O}_{2}$ es pequeña $\left(10^{-10}-10^{-9} \mathrm{M}\right)^{37}$, cantidades excesivas se generan en condiciones fisiopatológicas que se acompañan de estrés oxidativo como en la inflamación y en la reoxigenación postisquemia ${ }^{38}$. Si las peroxidasas, y otras hemoproteínas como la hemoglobina ${ }^{39,40}$ y la mioglobina ${ }^{13,14}$ peroxidan a FTZ, el sistema cit $\mathrm{c} / \mathrm{H}_{2} \mathrm{O}_{2}$ complementado con FTZ, sería un potencial generador de radicales libres catiónicos, los cuales pueden actuar sobre diversos blancos, como la TR y la LADH de $T c$, produciendo modificaciones que podrían determinar la acción tripanocida y otros efectos farmacológicos de las FTZ. El cit c es fácilmente liberado de las mitocondrias lesionadas y tendría acciones peroxidativas no sólo a nivel mitocondrial sino también en sitios extramitocondriales ${ }^{14}$. Los objetivos de este estudio son demostrar que el cit c, en presencia de peróxido de hidrógeno, oxida a las FTZ y determinar si los sistemas cit $\mathrm{c} / \mathrm{H}_{2} \mathrm{O}_{2} /$ FTZ actúan como inhibidores de la LADH de $T$ $c$. En este sentido, el estudio se orienta a los siguientes aspectos: a) Efecto de estos sistemas oxidativos sobre la actividad LADH de $T c$ y la relación estructura -actividad de las FTZ. b) Acción de los antioxidantes-interceptores de radicales libres- como blancos competitivos y protectores de la LADH. Además, como las diferencias estructurales entre la enzima del parásito y la LADH de mamífero ${ }^{17}$ han fundamentado la propuesta del diseño de inhibidores específicos de la enzima del $T c$, en este trabajo comparamos la inactivación de ambas enzimas por los sistemas cit $\mathrm{c} / \mathrm{H}_{2} \mathrm{O}_{2} / \mathrm{FTZ}$.

\section{MATERIAL Y MÉTODOS}

Enzimas y Reactivos: LADH de $T c$ fue obtenida por expresión en E. coli JRG 1342 (17). La enzima se mantuvo a $-20^{\circ} \mathrm{C}$ en fosfatos $(\mathrm{K})$ $100 \mathrm{mM}, \mathrm{KCl} 100 \mathrm{mM}$, EDTA $1.0 \mathrm{mM}$, pH 7.0, conteniendo glicerol al 50\% (v/v). La actividad específica fue de $\sim 400 \mathrm{U} / \mathrm{mg}$ de proteína. Esta preparación, que permaneció estable durante por lo menos un año, se diluyó al $25 \%(\mathrm{v} / \mathrm{v})$ en fosfatos (K) $50 \mathrm{mM}, \mathrm{pH} 7,4$ y se conservó a $4{ }^{\circ} \mathrm{C}$ para los experimentos. LADH de miocardio porcino y citocromo c de miocardio bovino; las fenotiazinas (FTZ), guayacol, GSH, Cys, Met, NAC, PAM, CPT, Tyr, TyrGly, (TyrCys) Trp, NADH, y ácido ascórbico se obtuvieron de Sigma Chemical Co. St. Louis, MO, USA. PZ-SO, CPZ-
SO y PMTZ-SO se prepararon por oxidación de la respectiva $\mathrm{FTZ}^{41}$ y fueron caracterizadas por cromatografía en capa delgada, por espectrofotometría midiendo la absorbancia a $341 \mathrm{~nm}(\mathrm{PZ}$ y CPZ sulfóxidos, $\varepsilon=6,3$ y $5,9 \mathrm{mM}^{-1} \mathrm{~cm}^{-1}$, respectivamente) ó $335 \mathrm{~nm}$ (PMTZ, $\varepsilon=5,0 \mathrm{mM}$ ${ }^{1} \mathrm{~cm}^{-1}$ ), y por fluorometría (espectros de emisión, con excitación de $325 \mathrm{~nm}$ ). Otros reactivos fueron iguales a los utilizados previamente ${ }^{14}$.

Actividad peroxidasa del citocromo c: Se determinó con guayacol (2-metoxifenol) como donante de hidrógeno ${ }^{42}$. En general, esta actividad fue medida a $30^{\circ} \mathrm{C}$, con guayacol $13 \mathrm{mM}$, cit c $5 \mu \mathrm{M}$, fosfatos (K) $50 \mathrm{M}, \mathrm{pH} 7.4, \mathrm{y} \mathrm{H}_{2} \mathrm{O}_{2} 0,5$ $\mathrm{mM}$. La reacción se inició con la adición del peróxido. El tetraguayacol, producto de la reacción, se midió por espectrofotometría a 470 $\mathrm{nm}$ hasta 60 min de incubación $\left(\varepsilon=26,6 \mathrm{mM}^{-1}\right.$ $\left.\mathrm{cm}^{-1}\right)$. Se requieren $4 \mu$ moles de $\mathrm{H}_{2} \mathrm{O}_{2}$ para generar $1 \mu \mathrm{mol}$ de tetraguayacol. La actividad peroxidasa del cit c se calculó con los valores de absorbancia de la parte lineal de la respectiva curva de formación del tetraguayacol. El efecto de la concentración de $\mathrm{H}_{2} \mathrm{O}_{2}$ y de cit c sobre la actividad peroxidasa se estudió variando las concentraciones respectivas, según lo indicado en resultados. Luego, la actividad peroxidasa del cit c se exploró con PZ y PMTZ como sustratos, midiendo la producción de PZ-SO y PMTZ-SO. Esta determinación se fundamenta en que la oxidación de las FTZ por sistemas peroxidasa $/ \mathrm{H}_{2} \mathrm{O}_{2}$ genera el radical catiónico $\mathrm{FTZ}^{+.14,40,43,44}$ el cual por una reacción no enzimática de dismutación (Reacción 2) produce el sulfóxido correspondiente con regeneración de la FTZ original ${ }^{39,40,45}$.

$2 \mathrm{FTZ}^{+}+\mathrm{H}_{2} \mathrm{O} \rightarrow \mathrm{FTZ}-\mathrm{SO}+\mathrm{FTZ}+2 \mathrm{H}^{+}(2)$

Las condiciones experimentales para medir la actividad peroxidasa y el efecto de la concentración de cit c ( con PZ) o del GSH (con PMTZ), se indican en resultados (Figuras 4 y 5, leyendas).

Inactivación de la LADH: La mezcla de inactivación contenía LADH, cit c, $\mathrm{H}_{2} \mathrm{O}_{2}$ FTZ, fosfatos (K) $50 \mathrm{mM}, \mathrm{pH} \mathrm{7,4}$ y otras adiciones como se indica en resultados. Además, esta mezcla contenía $\mathrm{KCl}$ 1,25 mM, EDTA 12,5 $\mu \mathrm{M}$ y glicerol $82 \mathrm{mM}$, resultado de la dilución de la preparación original de la LADH de $T c$. Muestras 
control, sin cit c o sin otros componentes de la mezcla de inactivación se estudiaron simultáneamente. La incubación de las muestras (volumen final $0,1 \mathrm{ml}$ ) fue a $30^{\circ} \mathrm{C}$ durante los tiempos indicados en cada experimento. Para medir la actividad LADH, se transfirieron alícuotas de $10 \mu \mathrm{l}$ a la mezcla de ensayo.

Actividad LADH: La actividad enzimática se midió a $30^{\circ} \mathrm{C}$. por la velocidad de oxidación del NADH, usando lipoamida como aceptor de electrones. La mezcla de ensayo contenía fosfatos(K) $50 \mathrm{mM}, \mathrm{pH}$ 7.4, $10 \mu \mathrm{l}$ de la mezcla de inactivación, lipoamida $1 \mathrm{mM}$ y NADH 0,2 $\mathrm{mM}$; volumen total 3,0 ml.

Determinación del radical $\mathbf{P Z}^{+\cdot}$ y de $\mathbf{F T Z}$ SO: La concentración del radical $\mathrm{PZ}^{+}$se determinó espectrofotométricamente utilizando el $\varepsilon_{518}=7,71 \mathrm{mM}^{-1} \mathrm{~cm}^{-1}$. La mezcla de reacción contenía fosfatos (K) $50 \mathrm{mM}, \mathrm{pH} 6.5$, cit c 5 ó $10 \mu \mathrm{M}, \mathrm{PZ} 100 \mu \mathrm{M}, \mathrm{H}_{2} \mathrm{O}_{2}$ 0,5 mM. La reacción se inició con la adición del peróxido de hidrógeno, midiéndose luego el $\Delta$ de absorbancia a $518 \mathrm{~nm}$ durante 10 ó más min.

La concentración de FTZ-SO se midió espectrofotométricamente en la mezcla de reacción que contenía lo indicado para la medida del radical $\mathrm{PZ}^{+}$, excepto la FTZ indicada. $\mathrm{La}$ determinación del $\Delta$ de absorbancia y los coeficientes de extinción, $\varepsilon^{40,41}$ se indicaron anteriormente.

Los espectros de emisión de fluorescencia se registraron en el espectrofluorómetro Aminco SLM 8000 C, con $325 \mathrm{~nm}$ de excitación, a 1, 10, 20 ó más min de incubación, en la mezcla de reacción como para la determinación espectrofotométrica, excepto que el volumen total fue de 2,0 $\mathrm{ml}$. El espectro de emisión de los estándares de PZ-SO, CPZ-SO y PMTZ-SO se registraron en los experimentos correspondientes.

Expresión de Resultados: La inactivación de la LADH por el sistema cit $\mathrm{c} / \mathrm{H}_{2} \mathrm{O}_{2} / \mathrm{FTZ}$, I(\%); la protección de la enzima, $\mathrm{P}(\%)$; los valores presentados y el análisis estadístico se expresan de acuerdo a lo indicado previamente ${ }^{14}$.

\section{RESULTADOS}

Actividad Peroxidasa del citocromo c.: La actividad peroxidasa fue de $1,8 \pm 0,10 \mu \mathrm{M}$ de
$\mathrm{H}_{2} \mathrm{O}_{2}$ consumido por min/mmol de cit c, utilizando como sustratos guayacol $13 \mathrm{mM} \mathrm{y} \mathrm{H}_{2} \mathrm{O}_{2} 2,5 \mathrm{mM}$. Esta actividad es baja comparada con la actividad peroxidasa de la $\mathrm{Mb}$ que, determinada en las mismas condiciones, fue de $21 \pm 2$ mmoles de peróxido de hidrógeno consumido por $\mathrm{min} / \mu \mathrm{mol}$ de $\mathrm{Mb}$. Como la actividad peroxidasa depende de la unión del peróxido al Fe del grupo hemo, la reducida actividad del cit $\mathrm{c}$ ha sido explicada por la hexacoordinación del $\mathrm{Fe}$ del hemo y el escaso acceso del $\mathrm{H}_{2} \mathrm{O}_{2}$ al Fe ${ }^{19,23,46}$. El sexto ligando $\mathrm{Met}^{80}$ puede ser desplazado por diversos factores y se ha propuesto que el peróxido puede desplazar al ligando Met con formación del radical ferrilporfirina y rápida oxidación de un residuo de tirosina ${ }^{23}$.

La Figura 2 muestra la cinética de la formación del producto de la acción peroxidasa del cit c, con una fase inicial de activación $<$ de 10 min, cuya duración disminuye con el aumento de la concentración de $\mathrm{H}_{2} \mathrm{O}_{2}$; luego, una fase "estable" representada por la parte recta de la curva y, finalmente, una zona con disminución notable de la formación del producto. Estas tres fases se indican en la Figura 2 como a (activación), b (fase estable) y c (inactivación) para la reacción cit c/guayacol en presencia de $\mathrm{H}_{2} \mathrm{O}_{2}$ 1,5 mM. Esta última parte de la curva ha sido explicada, por lo menos en parte, por la inactivación del catalizador ${ }^{34,47}$. La velocidad de la reacción del citocromo c en el estado "estable" tiene dependencia lineal de la concentración de $\mathrm{H}_{2} \mathrm{O}_{2}$ y aún a $20 \mathrm{mM}$ de peróxido no se observó saturación (resultados no mostrados), dependencia similar a la encontrada para el cit c de miocardio equino (47). El inserto de la Figura 2 muestra que la duración de la fase de activación también disminuye con el aumento de la concentración del cit c, mientras que la velocidad de la peroxidación aumenta. Considerando estos resultados hemos usado la concentración de cit c $5 \mu \mathrm{M}$ y $\mathrm{H}_{2} \mathrm{O}_{2} 0,5 \mathrm{mM}$, para prevenir la inactivación del cit c dependiente del peróxido y lograr una mayor duración de la fase lineal de la actividad peroxidasa.

FTZ como sustratos del sistema cit $\mathbf{c} / \mathrm{H}_{2} \mathrm{O}_{2}$ : La Figura 3 A y B muestra que la PZ es sustrato del sistema cit $\mathrm{c} / \mathrm{H}_{2} \mathrm{O}_{2}$ determinando, espectrofotométricamente, el radical $\mathrm{PZ}^{+}$producto de la reacción, por el aumento de la absorbancia a 518 $\mathrm{nm}^{44}$. Como se puede apreciar en la Figura, la 


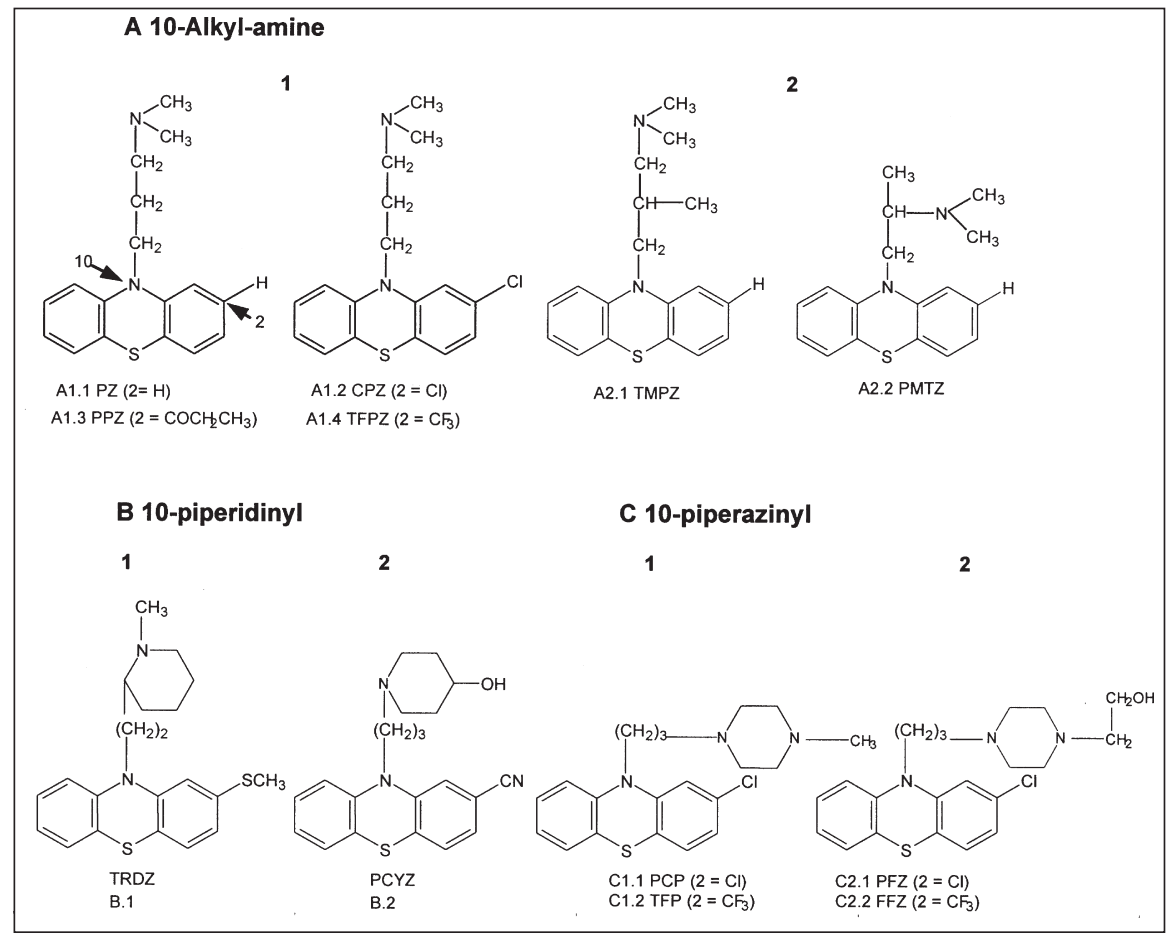

Figura 1. Clasificación y estructura de las fenotiazinas. Sustituyentes en posición 10: A. Con grupo alquilamina alifático (3-dimetilaminopropil o derivados) B. Con grupo piperidinil. C. Con grupo piperazinil. fase inicial de la formación de $\mathrm{PZ}^{+}$. corresponde a la fase de activación del cit c observada durante la reacción de peroxidación del guayacol (Figura 2). La cinética de producción de $\mathrm{PZ}^{+}$ resulta de dos reacciones opuestas: a) La peroxidación de $\mathrm{PZ}$ por el sistema cit $\mathrm{c} / \mathrm{H}_{2} \mathrm{O}_{2}$ (reacciones 3 y 4). b) La transformación del radical $\mathrm{PZ}^{+}$por dismutación principalmente (reacción 2). La Figura 3 A (curva GSH 0) y B (curva Tyr 0) muestran la relativa estabilidad del radical $\mathrm{PZ}^{+.13,45,48}$. La Figura 4 muestra que la $\mathrm{PZ}$ es sustrato del sistema cit $\mathrm{c} / \mathrm{H}_{2} \mathrm{O}_{2} \mathrm{mM}$, midiendo el PZ-SO resultante de la dismutación del radical $\mathrm{PZ}^{+}$, producto de la reacción peroxidasa ${ }^{13,40}$. En la misma Figura 4 se observa que la velocidad de la reacción se duplica (de $35 \mu \mathrm{M}$ a $68 \mu \mathrm{M}$ de PZSO formado en 30 min de incubación) al duplicar la concentración de cit c (de $5 \mu \mathrm{M}$ a $10 \mu \mathrm{M}$ ) y que la fase de activación es de menor duración cuando la concentración de cit c aumenta. Experimentos similares demostraron la formación de sulfóxidos con TRDZ, CPZ, PPZ, TFP y TFPZ en presencia de cit $\mathrm{c} / \mathrm{H}_{2} \mathrm{O}_{2}$ (comunicación personal).

La Figura 5 muestra la cinética de formación del producto de la reacción peroxidasa con PMTZ, diferente a la de la PZ, con un período de activación breve y rápida producción de PMTZSO, obteniéndose en $<$ de $8 \min 75 \mu \mathrm{M}$ de PMTZ y luego detención de la formación del producto. La Figura 5 también muestra que la ausencia de cit c previene la formación del sulfóxido, y que la adición de GSH cambia la cinética de la reacción, disminuyendo la producción de PMTZ$\mathrm{SO}$ en concordancia a lo observado anteriormente ${ }^{13}$, pues los tioles previenen la dismutación de los radicales FTZ $^{+}$. a FTZ-SO, debido a la reacción del tiol con el radical catiónico (reacción 5).

La Figura 6 y su inserto muestran los espectros de fluorescencia de los productos de peroxidación de PMTZ y PZ, respectivamente, por el sistema cit $\mathrm{c} / \mathrm{H}_{2} \mathrm{O}_{2}$, que se identificaron con el estándar del respectivo sulfóxido.

Inactivación de la LADH de $T$ c por sistemas cit $\mathbf{c} / \mathrm{H}_{2} \mathrm{O}_{2} /$ FTZ: La LADH fue inactivada por sistemas cit $\mathrm{c} / \mathrm{H}_{2} \mathrm{O}_{2} / \mathrm{FTZ}$ de acuerdo al tiempo de incubación y a la estructura de la FTZ. La Tabla 1 y la Figura 7 A y B, resumen el efecto de diferentes FTZ como sustratos del cit c/peróxido sobre la actividad LADH de $T c$. TRDZ y PZ constituyeron los más efectivos sistemas con $53 \%$ y $43 \%$ de inactivación de la 


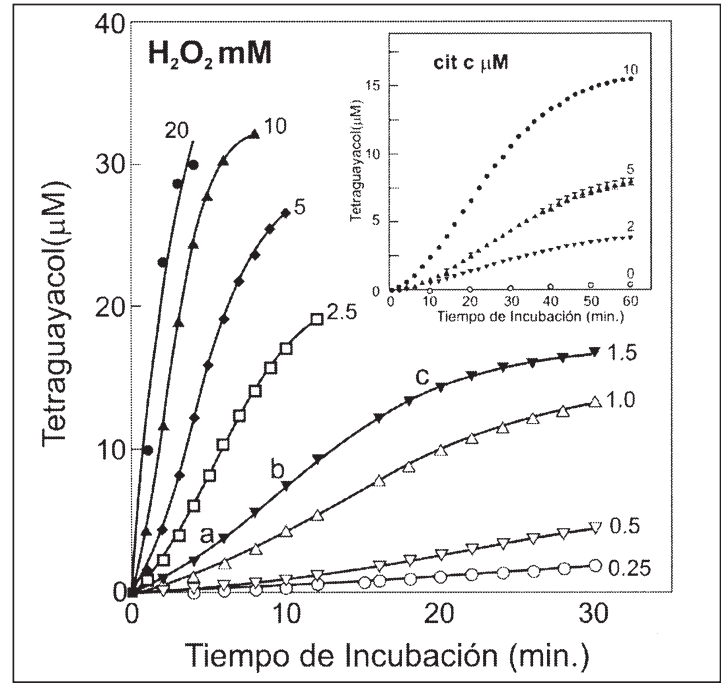

Figura 2. Actividad peroxidasa del citocromo c: Dependencia de la concentración del $\mathrm{H}_{2} \mathrm{O}_{2}$. La mezcla de ensayo contenía solución de fosfatos (K) $50 \mathrm{mM}$, pH 7.4 , guayacol $13 \mathrm{mM}$. cit. c $5 \mu \mathrm{M}$ y $\mathrm{H}_{2} \mathrm{O}_{2} 0.25-20 \mathrm{mM}$ según se indica en la Figura. La actividad peroxidasa se determinó midiendo el tetraguayacol formado. Los resultados corresponden a un experimento típico. En ausencia de peróxido de hidrógeno no se observó modificación del guayacol hasta los 30 min de incubación (no mostrados). Otras condiciones se describen en Material y Métodos. Inserto: Dependencia de la concentración de cit. c . La mezcla de reacción contenía solución de fosfatos (K) $50 \mathrm{mM}$, pH 7.4 , guayacol 13 $\mathrm{mM}$, cit. c .0, 2, 5 ó $10 \mu \mathrm{M}$ según se indica, $\mathrm{y}_{2} \mathrm{O}_{2} 0.5$ $\mathrm{mM}$. La actividad peroxidasa se determinó durante 60 min, midiendo el producto como en el experimento anterior.

LADH, respectivamente, después de 30 min de incubación. El efecto de estas dos FTZ fue similar después de 60 min de reacción (Tabla 1), pero al finalizar $90 \mathrm{~min}$ (Tabla 2, primera columna, y Figura 7 A y B) la PZ produjo mayor inactivación (82\%) que la TRDZ (76\%). TMPZ y CPZ tuvieron menor efecto $(72 \%$ y $68 \%$ de inactivación, respectivamente, a 90 min de incubación) (Tabla 2) Las otras FTZ fueron mucho menos eficaces, con inactivaciones entre $49 \%$ (PCP) y 15\% (PCYZ), después de 60 min de incubación (Tabla 1). La omisión de FTZ en la mezcla de reacción evitó la inactivación de la $\mathrm{LADH}$, lo cual descarta una acción directa del cit c/ $\mathrm{H}_{2} \mathrm{O}_{2}$ sobre la enzima del parásito. Así mismo, la exclusión del cit c de la mezcla de reacción previno el efecto inactivante, indicando la necesidad de cit c como catalizador de la

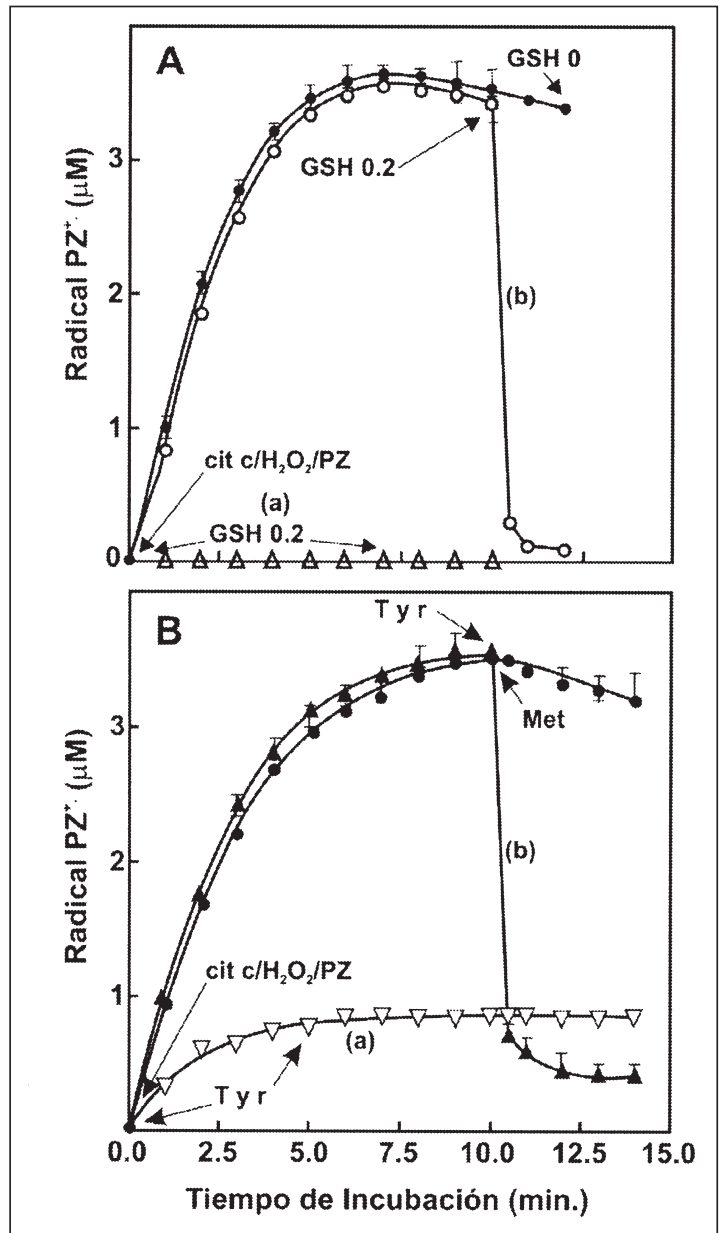

Figura 3. Producción de radical $\mathrm{PZ}^{+}$. por el sistema cit c/ $\mathrm{H}_{2} \mathrm{O}_{2} / \mathrm{PZ}$. A. Efecto del GSH. La mezcla de reacción contenía fosfatos (K) $6.5 \mathrm{mM}$, PZ $100 \mu \mathrm{M}$, cit c 5.0 $\mathrm{mM}, \mathrm{H}_{2} \mathrm{O}_{2} 0.5 \mathrm{mM}$, en ausencia (GSH 0) o presencia de GSH $0.2 \mathrm{mM}$ (a). A una de las muestras sin GSH se añadió el tiol (GSH $0.2 \mathrm{mM}$ ) a los $10 \mathrm{~min}$. de incubación (b) como se indica en la Figura. B. Efecto de la Tirosina y Metionina. Mezcla de reacción como en la Figura 3 A, excepto que en lugar de GSH se añadió Tyr $0.2 \mathrm{mM}$ (b) o Met $0.2 \mathrm{mM}$ a $10 \mathrm{~min}$. de incubación ; ó Tyr $0.2 \mathrm{mM}$ (a) un minuto antes del inicio de la reacción. Otras condiciones se describen en Material y Métodos.

peroxidación en los sistemas estudiados. Por otra parte, cuando la PZ fue sustituída por PZ-SO 100 $\mu \mathrm{M}$, en la mezcla de reacción, la inactivación de la LADH fue de $6 \pm 1 \%$ después de $90 \mathrm{~min}$ de incubación, lo cual indica ausencia de efecto del sulfóxido/cit $\mathrm{c} / \mathrm{H}_{2} \mathrm{O}_{2}$ sobre la actividad de ésta enzima. En un trabajo previo ${ }^{13}$, la PZ-SO $100 \mu \mathrm{M}$ en un sistema peroxidásico no mostró efecto 


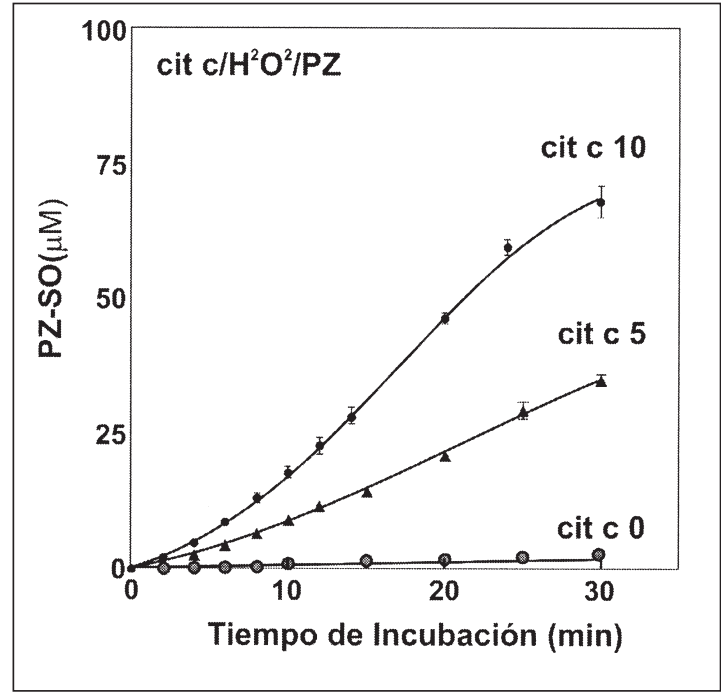

Figura 4. Producción de PZ-SO por el sistema cit. c/ $\mathrm{H}_{2} \mathrm{O}_{2} / \mathrm{PZ}$. La mezcla de reacción contenía fosfatos (K) 50 $\mu \mathrm{M}, \mathrm{pH}$ 6.5, PZ $100 \mu \mathrm{M}$, cit. c 0,5 ó $10 \mu \mathrm{M}$ según se indica en la Figura, y $\mathrm{H}_{2} \mathrm{O}_{2} 0.5 \mathrm{mM}$. La producción de PZ-SO se midió por el aumento de la absorbancia a 341 $\mathrm{nm}$. El tiempo de incubación se indica en la abscisa. Otras condiciones en Material y Métodos.

inactivante sobre la TR de $T c$, mientras que la PZ $10 \mu \mathrm{M}$, en un medio similar, inactivó completamente a esa enzima en $15 \mathrm{~min}$.

La relación estructura-actividad muestra que a pesar de las diferencias estructurales, la PZ (con grupo dimetilamino en $\mathrm{C}_{3}$ de la cadena propil en la posición N 10) y la TRDZ (con grupo piperidinil en $\mathrm{N} 10$ y $\mathrm{SCH}_{3}$ en la posición 2) poseen el más alto efecto inactivante de la $\mathrm{LADH}$, mientras que la PMTZ, con mayor parecido estructural a la PZ (posee el grupo dimetilamino en $\mathrm{C}_{2}$ de la cadena propil en $\mathrm{N} 10$ )tiene menor efecto inactivante, $47 \%$, sobre la $\mathrm{LADH}$, que representa el 57\% del nivel alcanzado con la PZ. CPZ, PCP y PFZ, estructuralmente relacionadas por tener $\mathrm{Cl}$ en la posición 2 del núcleo de la FTZ, mostraron mayor efecto inactivante sobre la LADH que los compuestos con el grupo $\mathrm{CF}_{3}$ en la posición 2 del núcleo, FFZ, TFP y TFPZ. (Tabla 1). Estas últimas, y también la PPZ y PCYZ (Figura 7, A y B) tuvieron un período de latencia más prolongado antes de ejercer su efecto inactivante.

Protección de la LADH de $T \boldsymbol{c}$ frente a los radicales FTZ $^{+\cdot}$ : La Tabla 3 muestra que los compuestos tiol previnieron totalmente la inactivación de la LADH hasta los 30 minutos de

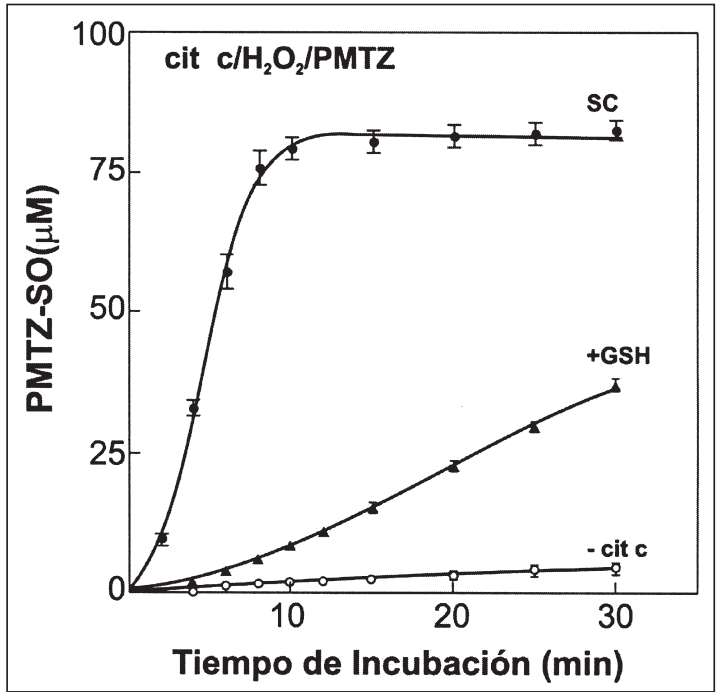

Figura 5. Producción de PMTZ-SO por el sistema cit. c / $\mathrm{H}_{2} \mathrm{O}_{2} / \mathrm{PMTZ}$. La mezcla de reacción contenía fosfatos $(\mathrm{K})$ $50 \mathrm{mM}$, pH 6.5, PMTZ $100 \mu \mathrm{M}$, cit. c $5 \mathrm{mM}, \mathrm{H}_{2} \mathrm{O}_{2} 0.5$ $\mathrm{mM}$ (SC, sistema completo) y GSH $0.2 \mathrm{mM}$, según se indica en la Figura. La muestra control no contenía cit.c (-cit. c). La PMTZ-SO se midió por el aumento de la absorbancia a 335 $\mathrm{nm}$ El tiempo de incubación se indica en la abscisa. Otras condiciones se describen en Material y Métodos.

incubación, pero después de $60 \mathrm{~min}$. la protección por GSH y PAM fue sólo parcial, $64 \%$ y $74 \%$ respectivamente. NAC y $\mathrm{CPT}$ fueron más efectivos con $100 \%$ y $93 \%$ de protección a los $60 \mathrm{~min}$. Entre los compuestos tiol, el GSH presentó el menor efecto protector, $16 \%$ a los 90 min de incubación (Figura 8). La adición de GSH $0,2 \mathrm{mM}$ a una muestra de LADH de $T c$, inactivada durante $60 \mathrm{~min}$ por el sistema cit c/ $\mathrm{H}_{2} \mathrm{O}_{2} / \mathrm{PZ}$, según el procedimiento descrito en la Tabla 3, no restauró la actividad de la LADH lo que sustenta el efecto inactivante irreversible de los radicales $\mathrm{PZ}^{+-}$. En la Tabla 3 se observa que la tirosina y sus péptidos poseen notable efecto protector, mientras que el triptofano efecto sólo fue discreto $(20 \%$ y $11 \%$ de proteción después de 30 y $60 \mathrm{~min}$, respectivamente). La Tabla 3 también muestra que el NADH previno totalmente la inactivación de la LADH por el mismo sistema peroxidativo después de $30 \mathrm{~min}$ de incubación, pero este efecto fue mucho menor, $21 \%$, (Tabla 3 ) a los 60 min y $9 \%$ a los 90 min de incubación (Figura 8). Ascorbato y trolox fueron los mejores protectores de la LADH, como en el estudio con el sistema $\mathrm{MPO} / \mathrm{H}_{2} \mathrm{O}_{2} / \mathrm{TRDZ}$ (14). Para los experimentos de protección en el presente estudio 


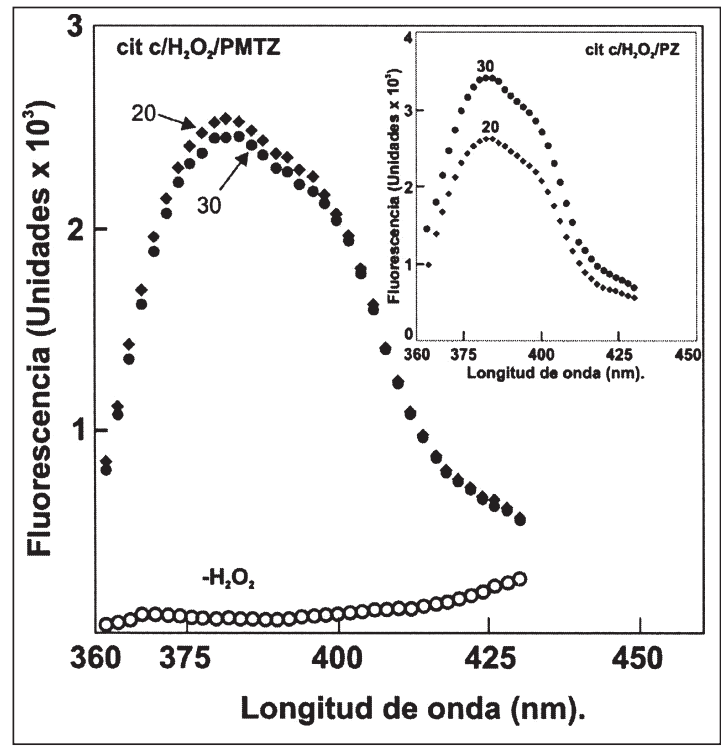

Figura 6. Espectros de emisión de fluorescencia del producto de oxidación de la PMTZ por el sistema cit. c/ $\mathrm{H}_{2} \mathrm{O}_{2}$. La mezcla de reacción contenía fosfatos $(\mathrm{K}) 50$ $\mathrm{mM}, \mathrm{pH}$ 6.5, PMTZ $100 \mu \mathrm{M}$, cit. c $5 \mu \mathrm{M}, \mathrm{H}_{2} \mathrm{O}_{2} 0.5$ $\mathrm{mM}$. La muestra control no contenía $\mathrm{H}_{2} \mathrm{O}_{2}$. Los espectros de emisión de fluorescencia se registraron a 20 y 30 minutos de incubación con excitación de $325 \mathrm{~nm}$. Otro control PMTZ/ $\mathrm{H}_{2} \mathrm{O}_{2}$ (cit. c omitido) no presentó este espectro (no mostrado).El espectro de emisión del producto de la oxidación de la PMTZ por cit. c/ $\mathrm{H}_{2} \mathrm{O}_{2}$ presenta la máxima fluorescencia a $382 \mathrm{~nm}$ como el espectro de emisión de la PMTZ-SO. Otras condiciones se describen en Material y Métodos. Inserto: Espectros de emisión del producto de oxidación de la $\mathrm{PZ}$ por el sistema cit.c/ $\mathrm{H}_{2} \mathrm{O}_{2}$ a 20 y 30 min de incubación y del control sin $\mathrm{H}_{2} \mathrm{O}_{2}$ a 30 min, obtenidos con excitación de $325 \mathrm{~nm}$. Los espectros del sistema completo tienen características del espectro de emisión de la PZ-SO, con la máxima fluorescencia a 384 $\mathrm{nm}$.

se usó PZ, pues fue la FTZ con mayor efecto inactivante y por que la velocidad de transformación del radical $\mathrm{PZ}^{+}$es relativamente lenta (Figura 3 A y B) $)^{14,45,48}$.

\section{Protectores de la LADH como supresores} de radicales FTZ ${ }^{+}$: La Tabla 4 muestra que los compuestos GSH, Cys, NAC, Tyr y Trp determinaron rápida disminución de la concentración del radical $\mathrm{PZ}^{+}$generado por el cit c activado. La Figura 3 A (b) y B (b) también muestran que el GSH y la tirosina, respectivamente, produjeron rápida caída del nivel de $\mathrm{PZ}^{+}$. cuando se añadieron a los 10 min de iniciada la reacción de la fenotiazina con cit $\mathrm{c} / \mathrm{H}_{2} \mathrm{O}_{2}$, pero la $\mathrm{L}$

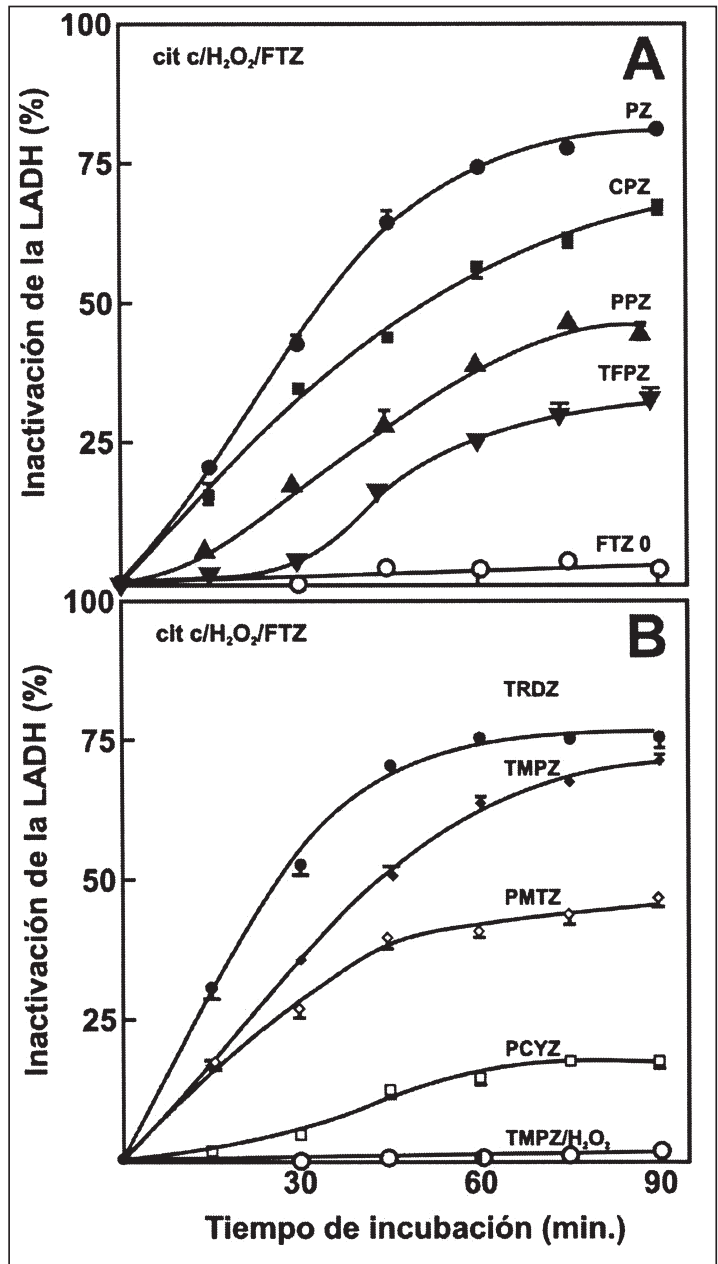

Figura 7. Cinética de la inactivación de la LADH de $T$. cruzi por los sistemas cit $\mathrm{c} / \mathrm{H}_{2} \mathrm{O}_{2} / \mathrm{FTZ}$. La mezcla de

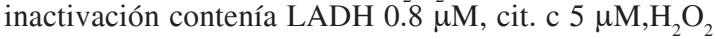
$0.5 \mathrm{mM}$, fosfatos (K) $50 \mathrm{mM}$, pH 7.4, y FTZ $100 \mu \mathrm{M}^{2}$ según se indica: A Fenotiazinas con grupo 3- dimetilamino-propil en N 10 (PZ,CPZ,PPZ y TFPZ). B. Con grupos 2-metil-3-dimetil- amino propil (TMPZ) o 2dimetil-amino-propil (PMTZ); o con grupos piperidinil (TRDZ o PCYZ). Tiempo de incubación como se indica en la abscisa. Los valores representan la media \pm D.E. $(n=3-5)$. Los controles en ausencia de FTZ o en ausencia de cit. c para el sistema TMPZ/ $\mathrm{H}_{2} \mathrm{O}_{2}$, se presentan en A y B, respectivamente. La omisión de cit. c para los sistemas con las otras FTZ, o la omisión de $\mathrm{H}_{2} \mathrm{O}_{2}$, también previnieron la inactivación de la enzima (no mostrados).

Metionina no causó significativa variación de la concentración de $\mathrm{PZ}^{+}$. (Figura 3B, Met) Las Figuras 3 A (a) y B (a) muestran que el GSH y la tirosina, respectivamente, presentes desde un minuto antes del inicio de la reacción, tuvieron 
Tabla 1. Inactivación de la LADH de Trypanosomac cruzi por el sistema cit $\mathrm{c} / \mathrm{H}_{2} \mathrm{O}_{2} /$ FTZ

\begin{tabular}{lcr}
$\begin{array}{l}\text { FTZ } \\
(\mathbf{1 0 0} \boldsymbol{\mu M})\end{array}$ & \multicolumn{2}{c}{ Inactivación de la LADH $(\mathbf{\%})$} \\
& $\begin{array}{c}\text { Tiempo de Incubación: } \\
\text { 30 min }\end{array}$ & $\mathbf{6 0}$ min \\
\hline PZ & $43 \pm 1$ & $75 \pm 1$ \\
TRDZ & $53 \pm 2$ & $76 \pm 1$ \\
TMPZ & $36 \pm 1$ & $64 \pm 1$ \\
CPZ & $35 \pm 1$ & $57 \pm 2$ \\
PCP & $36 \pm 1$ & $49 \pm 2$ \\
PFZ & $32 \pm 1$ & $41 \pm 1$ \\
PMTZ & $27 \pm 4$ & $41 \pm 5$ \\
PPZ & $18 \pm 1$ & $40 \pm 1$ \\
FFZ & $16 \pm 1$ & $35 \pm 1$ \\
TFP & $17 \pm 1$ & $31 \pm 1$ \\
TFPZ & $0 \pm 0$ & $25 \pm 3$ \\
PCYZ & $5 \pm 0$ & $15 \pm 1$ \\
Ninguna & $0 \pm 0$ & $3 \pm 1$ \\
\hline
\end{tabular}

La mezcla de inactivación contenía fosfatos $(\mathrm{K}) 50 \mathrm{mM}$, pH 7.4, LADH $0.8 \mu \mathrm{M}$, cit c $5 \mu \mathrm{M}, \mathrm{H}_{2} \mathrm{O}_{2} 0.5 \mathrm{mM}, \mathrm{FTZ}$ $100 \mu \mathrm{M}$. Tiempo de incubación como se indica en la Tabla. Los valores presentados son la media \pm D. E. de 3-5 determinaciones. La inactivación de la LADH (\%) por los sistemas $\mathrm{H}_{2} \mathrm{O}_{2} / \mathrm{FTZ}$ ( cit.c omitido) fue de 0-4\% (resultados no mostrados). Otras condiciones experimentales según Material y Métodos.

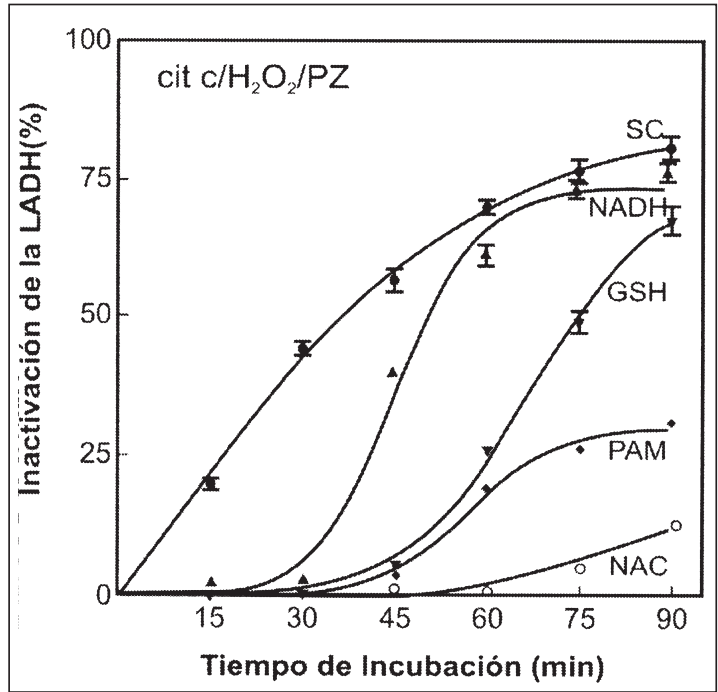

Figura 8. Efecto de compuestos tiol y del NADH sobre la inactivación de la LADH de T. Cruzi por el sistema cit.c/ $\mathrm{H}_{2} \mathrm{O}_{2} / \mathrm{PZ}$. Condiciones experimentales en la leyenda de la Figura 7, con PZ $100 \mu \mathrm{M}$ (SC, sistema completo). La mezcla de inactivación contenía, además, GSH, PAM, NAC o NADH $0.2 \mathrm{mM}$ según se indica en la Figura. La reacción se inició añadiendo $\mathrm{H}_{2} \mathrm{O}_{2}$. El control de $\mathrm{SC}\left(\mathrm{H}_{2} \mathrm{O}_{2}\right.$ omitido) no tuvo efecto sobre la actividad de la enzima durante el experimento. (resultados no mostrados). Tiempo de incubación se indica en la abscisa. Otras condiciones según se describe en Material y Métodos. Los valores de $\mathrm{P}$ fueron $<0,001$ (NAC y PAM) $,<0,005$ (GSH) y < 0,01 (NADH), a los 90 min de incubación.

Comparación entre la inactivación de la LADH de $T \boldsymbol{c}$ y de mamífero: La probable diferencia entre la inactivación de la LADH del parásito y la del huésped, fue estudiada comparando la interacción entre la LADH del $T c$ y la enzima de mamífero con varios sistemas cit

Tabla 2 Inactivación de la LADH de Trypanosoma cruzi y de miocardio por sistemas cit c/H2O2/FTZ

\begin{tabular}{|c|c|c|c|}
\hline \multirow{2}{*}{$\begin{array}{l}\text { FTZ } \\
(100 \mu M)\end{array}$} & \multicolumn{3}{|c|}{ Inactivación de la LADH ( \%) } \\
\hline & T cruzi & Miocardio & $\begin{array}{c}\text { Relación } \\
\text { T cruzi/miocardio }\end{array}$ \\
\hline $\mathrm{PZ}$ & $82 \pm 1$ & $45 \pm 1,5$ & 1,8 \\
\hline TRDZ & $76 \pm 2$ & $40 \pm 1,5$ & 1,9 \\
\hline TMPZ & $72 \pm 1$ & $34 \pm 0,5$ & 2,1 \\
\hline $\mathrm{CPZ}$ & $68 \pm 1$ & $25 \pm 1,0$ & 2,7 \\
\hline PMTZ & $47 \pm 3$ & $14 \pm 1,0$ & 3,3 \\
\hline
\end{tabular}

La mezcla de inactivación contenía LADH de T. cruzi $0.8 \mu \mathrm{M}$ o de miocardio $1.0 \mu \mathrm{M}$; cit c $5 \mu \mathrm{M}$; $\mathrm{H} 2 \mathrm{O} 20.5 \mathrm{mM}$; FTZ $100 \mu \mathrm{M}$; y fosfatos (K) $50 \mathrm{mM}, \mathrm{pH}$ 7,4. Tiempo de incubación $90 \mathrm{~min}$. Otras condiciones experimentales según Material y Métodos. Los valores representan la media \pm D. E. $(n=3)$ de la inactivación de la LADH $(\%)$. 
Tabla 3. Protección de la LADH de Trypanosoma cruzi por compuestos tiol, aminoácidos y otros antioxidantes frente al sistema cit c $/ \mathrm{H}_{2} \mathrm{O}_{2}$

\begin{tabular}{|c|c|c|}
\hline \multirow[t]{2}{*}{ Antioxidantes } & \multicolumn{2}{|c|}{ Inactivación de la LADH (\%) } \\
\hline & Incubación: $30 \mathrm{~min}$ & $60 \mathrm{~min}$ \\
\hline Ninguno & $44 \pm 1$ & $73 \pm 2$ \\
\hline \multicolumn{3}{|l|}{ Compuestos Tiol } \\
\hline GSH & $1 \pm 1(98)$ & $26 \pm 2(64)$ \\
\hline NAC & $0 \pm 0(100)$ & $0 \pm 1(100)$ \\
\hline CPT & $0 \pm 1(100)$ & $5 \pm 2(93)$ \\
\hline PAM & $1 \pm 1(98)$ & $19 \pm 2(74)$ \\
\hline \multicolumn{3}{|c|}{ Aminoácidos y péptidos } \\
\hline Tyr & $16 \pm 2(64)$ & $40 \pm 2(45)$ \\
\hline TyrGly & $24 \pm 2(45)$ & $36 \pm 2(51)$ \\
\hline$(\text { TyrCys })_{2}$ & $11 \pm 3(75)$ & $25 \pm 1(66)$ \\
\hline $\operatorname{Trp}$ & $35 \pm 2(20)^{*}$ & $65 \pm 1(11)^{*}$ \\
\hline \multicolumn{3}{|c|}{ Otros antioxidantes } \\
\hline NADH & $0 \pm 0(100)$ & $58 \pm 2(21)$ \\
\hline Ascorbato & $1 \pm 1(98)$ & $9 \pm 2(88)$ \\
\hline Trolox & $2 \pm 0(95)$ & $0 \pm 0(100)$ \\
\hline
\end{tabular}

La mezcla de inactivación contenía LADH $0.8 \mu \mathrm{M}$, cit c $5 \mu \mathrm{M}, \mathrm{H}_{2} \mathrm{O}_{2} 0.5 \mathrm{mM}, \mathrm{PZ} 100 \mu \mathrm{M}$, fosfatos (K) $50 \mathrm{mM}$, pH 7.4 y antioxidante $0.2 \mathrm{mM}$ según se indica en el Cuadro. Los valores entre paréntesis indican la protección de la LADH (\%). Otras condiciones se describen en Material y Métodos. $\mathrm{P}<0,001$ para todos los sistemas, excepto los marcados con un asterisco $(* \mathrm{P}<0,005)$.

Tabla 4 .Efecto de los compuestos tiol, aminoácidos y otros antioxidantes sobre la concentración del radical $\mathrm{PZ}+$

\begin{tabular}{lc}
\hline Antioxidante & $\begin{array}{c}\text { Concentración de } \mathbf{P Z}^{+.} \\
\text {después de la incubación } \\
(\boldsymbol{\mu M})\end{array}$ \\
\hline Ninguno & $3,7 \pm 0,2$ \\
Compuestos tiol & \\
GSH & $0,1 \pm 0,1(96)$ \\
Cys & $0,7 \pm 0,1(81)$ \\
NAC & $0,0 \pm 0,0(100)$ \\
Aminoácidos & \\
Tyr & $0,6 \pm 0,2(84)$ \\
Trp & $0,2 \pm 0,1(95)$ \\
Otros antioxidantes & $0,2 \pm 0,1(95)$ \\
NADH & $0,2 \pm 0,1(95)$ \\
Ascorbato & $0,0 \pm 0,0(100)$ \\
Trolox &
\end{tabular}

La mezcla de reacción contenía cit c $5 \mu \mathrm{M}, \mathrm{H}_{2} \mathrm{O}_{2} 0.5 \mathrm{mM}$, PZ $0.1 \mathrm{mM}$, fosfatos $(\mathrm{K}) 50 \mathrm{mM}$, pH 6,5. La reacción se inició con la adición de $\mathrm{H}_{2} \mathrm{O}_{2}$ y a los 10 minutos de incubación se determinó por espectrofotometría la producción de $\mathrm{PZ}^{+}$, y de inmediato se añadió el antioxidante $0.2 \mathrm{mM}$. Después de un minuto adicional de incubación se midió nuevamente la concentración de $\mathrm{PZ}^{+}$ Entre paréntesis la disminución (\%) de la concentración de $\mathrm{PZ}^{+}$en comparación con la concentración previa del radical a la adición del antioxidante. $(\mathrm{P}<0,001$ en todos los casos). c/ $\mathrm{H}_{2} \mathrm{O}_{2} / \mathrm{FTZ}$. La Tabla 2 muestra que la inactivación de la LADH de $T c$ fue de 1,8, 1,9 y 2,1 veces la inactivación de la enzima de mamífero, con PZ, TRDZ y TMPZ, respectivamente. La CPZ y PMTZ presentaron efecto inactivante aún mayor frente a la LADH de $T c$, con una relación de efecto LADH $T c / \mathrm{LADH}$ de miocardio de 2,7 y 3,3 respectivamente.

\section{DISCUSIÓN}

Aquí demostramos que el cit c posee actividad peroxidasa (Figura 2 e inserto) y que las FTZ son sustratos de esa reacción (Figuras 3-6). Es bastante conocido que los productos de la actividad peroxidasa con FTZ son los radicales libres catiónicos, $\mathrm{FTZ}^{+13,14,39,40,43-45}$.

En este. estudio la formación del radical $\mathrm{PZ}^{+}$. fue seguida directamente por espectrofotometría (Figuras 3 A y B; Tabla 4) y por su conversión a PZ-SO (Figuras 4 y 6, inserto). La generación de sulfóxidos en los sistemas oxidativos empleados, ocurre previa formación de radicales FTZ $^{+}$, por lo cual la determinación cuantitativa e identificación de otros sulfóxidos como el de la PMTZ (Figuras 5 y 6) sustenta la peroxidación de estos sustratos por el sistema cit $\mathrm{c} / \mathrm{H}_{2} \mathrm{O}_{2}$. Entonces, el cit c por su acción peroxidativa es 
un generador de $\mathrm{FTZ}^{+}$Esta acción implica a) La formación de oxoferril cit c (reacción 3) que contiene radical tirosil ${ }^{23,26}$, estructuralmente similar al compuesto I de la HRP, de la hemoglobina y $\mathrm{Mb}^{39,49-51}$; y b) La generación de radicales $\mathrm{FTZ}^{+}$ (reacción 4).

cit c-Fe $\mathrm{FII}+\mathrm{H}_{2} \mathrm{O}_{2} \rightarrow$ cit c $^{+\cdot-}-\mathrm{Fe}^{\mathrm{IV}}=\mathrm{O}+\mathrm{H}_{2} \mathrm{O}$

cit $\mathrm{c}^{+} \cdot-\mathrm{Fe}^{\mathrm{IV}}=\mathrm{O}+\mathrm{FTZ}+\mathrm{H}^{+} \rightarrow$ cit $\mathrm{c}^{+.}-\mathrm{Fe}^{\mathrm{III}}+$ $\mathrm{FTZ}^{+}+\mathrm{OH}^{-}(4)$

El ferril cit c es la especie activa ${ }^{32,33}$ que puede iniciar la peroxidación de lípidos, y que oxidaría a diversas moléculas ${ }^{27-32,34,35}$, incluyendo FTZ con generación de radicales $\mathrm{FTZ}^{+}$.

En este trabajo se demuestra que las FTZ, previa oxidación a radicales libres $\mathrm{FTZ}^{+}$por el sistema cit $\mathrm{c} / \mathrm{H}_{2} \mathrm{O}_{2}$, reaccionan con la LADH de $T c$ inhibiéndola irreversiblemente (Tabla 1; Figura 7 A y B). El rol inactivante de los radicales FTZ se sustenta en: a) El requerimiento de un catalizador de la reacción peroxidasa, cit c en este estudio, y la participación del $\mathrm{H}_{2} \mathrm{O}_{2}$. Las FTZ (14) y los sistemas $\mathrm{H}_{2} \mathrm{O}_{2} / \mathrm{FTZ}$ y $\mathrm{H}_{2} \mathrm{O}_{2} /$ cit c carecieron de efecto inactivante significativo (Tabla 1; Figuras 7 A y B). b) La detección y determinación cuantitativa de $\mathrm{PZ}^{+}$. (Figuras 3 A y B) así como en la medida de los sulfóxidos derivados por dismutación de éstos radicales, (Figuras 4 y 5) y los espectros de emisión de fluorescencia de FTZSO resultantes de la reacción 2 (Figura 6 e inserto). c) El efecto protector del GSH, NAC, Cys, NADH y otros supresores de radicales libres, frente a la inactivación de la LADH por cit c/ $\mathrm{H}_{2} \mathrm{O}_{2} / \mathrm{PZ}$ (Tabla 3 , Figura 8). d) El efecto de los protectores de la LADH sobre los niveles de $\mathrm{PZ}^{+}$. (Tabla 4, Figuras 3 A y B ).

El efecto inactivante de las FTZ sobre la LADH de $T c$ fue en el siguiente orden: PZ>TRDZ $>$ TMPZ > CPZ > PCP > PFZ, PMTZ, PPZ. Las otras FTZ tuvieron discreto efecto (Tabla 1). La actividad del sistema cit $\mathrm{c} / \mathrm{H}_{2} \mathrm{O}_{2} / \mathrm{FTZ}$, como en otros sistemas peroxidásicos ${ }^{14}$ dependió de la estructura de las FTZ. PZ y TRDZ presentaron la mayor actividad inhibitoria y aunque son estructuralmente diferentes, el grupo sustituyente en la posición 10 de la TRDZ puede considerarse como un derivado cíclico del respectivo grupo de la PZ. La TMPZ presentó alto efecto inactivante y su grupo sustituyente en N 10 es análogo al de la PZ. Sin embargo, la PMTZ con una estructura bastante similar a PZ y TMPZ (Figura $1 \mathrm{~A}$ ) tuvo menor efecto inactivante (Tabla 1 y Figura 7 B), que parece depender del número de átomos de C ( PZ y TMPZ, n=3; PMTZ, $\mathrm{n}=2$ )entre el átomo de $\mathrm{N}$ del sustituyente y el $\mathrm{N}$ 10 del núcleo FTZ (Figura 1 A y Ref. 45). En la Figura 8 A se observa que el efecto inactivante sobre la LADH es en el orden:

\section{$\mathrm{PZ}>\mathrm{CPZ}>\mathrm{PPZ}>\mathrm{TFPZ}$}

en relación inversa con la propiedad de captar electrones inherente a los sustituyentes en la posición 2 del anillo heterocíclico de las FTZ. Así, la TFPZ que en la posición 2 tiene el grupo $\mathrm{CF}_{3}$ con fuerte poder de captación de electrones, presentó el menor efecto inactivante sobre la LADH (Figura 8 A). Esta propiedad de los sustituyentes en la posición 2 puede explicar el mayor efecto inactivante sobre la LADH de las FTZ con $\mathrm{Cl}$ en la posición 2 (Figura $1 \mathrm{~A}$ y $\mathrm{C}$ ) que las FTZ con $\mathrm{CF}_{3}$ en la misma posición (Tabla 1 y Figura $7 \mathrm{~A}$ ), así como el alto efecto inactivante de la TRDZ (Tablas 1 y 2: Figura 7 B), cuyo grupo tiometil en la posición 2 (Figura 1 B) tiene débil poder de atracción de electrones. La influencia de los sustituyentes sobre la actividad de las FTZ si bien puede depender del diferente acceso de estos sustratos al sitio hidrofóbico en que se encuentra el hemo del cit c, como en el caso de los sustratos de la $\mathrm{MPO}^{52}$, la estabilidad del radical catiónico es un factor determinante, la cual depende de la estructura de los sustituyentes en las posiciones 2 y $10^{45}$ y de la interacción de ambos con el núcleo de las FTZ ${ }^{48}$. El radical $\mathrm{PZ}^{+}$es más estable que los radicales $\mathrm{TRDZ}^{+.13}$, $\mathrm{CPZ}^{+}$(43) y $\mathrm{PMTZ}^{+.45}$, en concordancia con sus actividades frente a la LADH de $T c$ (Figura 7 A y B; $\operatorname{Ref}^{14}$ ).

Los compuestos tiol tienen efecto protector de la LADH frente al sistema cit $\mathrm{c} / \mathrm{H}_{2} \mathrm{O}_{2} / \mathrm{PZ}$ (Tabla 3, Figura 8), similarmente a lo observado en otros sistemas peroxidasas, MPO, HRP y $\mathrm{Mb}^{14}$. La reacción de los radicales $\mathrm{FTZ}^{+}$. con compuestos tiol ha sido anteriormente estudiada ${ }^{17,53}$ y su efecto protector confirma el rol de $\mathrm{FTZ}^{+}$en la inactivación de la LADH. El efecto del GSH como desactivante de radicales $\mathrm{PZ}^{+}$. (Figura $3 \mathrm{~A}$, línea (b) se explica porque el radical $\mathrm{PZ}^{+}$actúa como aceptor de un electrón del anión glutatión, formándose el radical glutatiilo y la molécula neutra PZ, a pH 
fisiológico o valores de $\mathrm{pH}$ aún mayores (reacción 5).

$\mathrm{GS}^{-}+\mathrm{PZ}^{+\cdot} \leftrightarrow \mathrm{GS}+\mathrm{PZ}(5)$

La reacción inversa de transferencia de un electrón de PZ a GS· solamente ocurre en medios de $\mathrm{pH}$ muy ácido. La producción de radicales tiílo potencialmente citotóxicos y el consumo de GSH, con el consiguiente desbalance redox y predominio de la forma oxidada, son mecanismos de la acción del GSH y otros compuestos tiol como supresores de radicales $\mathrm{FTZ}^{+}$. El efecto transitorio del GSH como protector de la LADH (Figura 8) puede explicarse, por lo menos en parte, por la concentración relativamente pequeña del GSH empleada y su consumo durante los primeros 45 min de incubación. El efecto inhibidor de la formación de radicales $\mathrm{PZ}^{+}$observado en la Figura 3 A línea (a) puede explicarse, en parte, por la competencia entre PZ y GSH por el cit c activado, pues recientemente se ha demostrado que el GSH es oxidado por el grupo oxoferril del compuesto I del cit $\mathrm{c}^{33}$.

La L Tirosina y sus péptidos, y el L Triptofano, fueron protectores de la LADH de $T c$ (Tabla 3). En concordancia con este efecto, la tirosina añadida un min. antes del inicio de la reacción, inhibió la producción del radical $\mathrm{PZ}^{+}$. (Figura 3 B, curva a), y cuando la tirosina se añadió al obtener la máxima concentración de $\mathrm{PZ}^{+}$, ésta disminuyó abruptamente (Tabla 4 y Figura 3B, línea b). El efecto inhibidor(a) puede explicarse por la demostración de que las FTZ (Figuras $3 \mathrm{~A}$ y B-6) y la tirosina son sustratos del cit c activado.En la reacción de la tirosina se forma eficientemente el radical tirosil (reaccion 6) (comunicación personal) determinado por la fluorescencia de su dímero, la ditirosina (reacción 7), como en la reacción catalizada por peroxidasas 54,55 .

$\mathrm{Tyr}+$ cit $^{+} \cdot-\mathrm{Fe}^{\mathrm{IV}}=\mathrm{O} \rightarrow \mathrm{Tyr}+\mathrm{cit}^{+} \cdot-\mathrm{Fe}^{\mathrm{III}}+\mathrm{OH}(6)$

Tyr + Tyr $\rightarrow$ Tyr-Tyr (7)

Entonces, FTZ y Tyr pueden ser sustratos competitivos del cit c en su reacción con el peróxido, con el resultado de la prevención de la peroxidación de las FTZ por la Tyr. El efecto supresor del radical $\mathrm{PZ}^{+}$. demostrado en la Figura 3B (b) puede ocurrir en la siguiente reacción:
$\mathrm{Tyr}+\mathrm{PZ}^{+} \rightarrow \mathrm{Tyr}+\mathrm{PZ}(8)$

Esto es, el radical $\mathrm{PZ}^{+}$generado por oxidación previa de la PZ por el cit c activado, oxida a un compuesto secundario (Tirosina) produciendo el respectivo radical (Tirosil).Esta interpretación se basa en la propiedad de las FTZ de actuar como mediadores redox ${ }^{56-58}$, por su carácter de oxidantes fuertes frente a compuestos reductores como los aminoácidos aromáticos Tyr y Trp. Por otra parte, la tirosina libre ${ }^{59} \mathrm{o}$ formando parte de proteínas de membrana ${ }^{60}$, o de péptidos hormonales $^{61}$ puede actuar como eficiente antioxidante fisiológico. Al respecto, el péptido TyrGly se comportó como protector de la LADH de $T c$ (Tabla 3), en concordancia con la propiedad antioxidante de polipéptidos $\mathrm{NH}_{2}$-TyrGly-como la Leu y Met encefalinas ${ }^{61}$.

En la reacción del cit c con el peróxido de hidrógeno se ha demostrado que después de la generación del radical centrado en la proteína del cit c, éste puede ser transferido a un nonapéptido que contiene triptofano y a otras moléculas ${ }^{26}$, y en relación al Trp se ha demostrado que el FSHRF-factor liberador de la hormona folículo estimulante- posee actividad antioxidante como interceptor de radicales libres, dependiente de los dos residuos de Trp de su molécula ${ }^{61}$. Entonces, el efecto protector del Trp hacia la LADH, podría explicarse por la acción competitiva del Trp frente a la PZ por el cit c activado, así como por el efecto supresor del radical $\mathrm{PZ}^{+}$por el Trp (Tabla 4); éste último efecto según la reacción:

$\operatorname{Trp}+\mathrm{PZ}^{+} \rightarrow \operatorname{Trp}+\mathrm{PZ}(9)$

estaría de acuerdo a los potenciales redox del par $\mathrm{PZ}^{+}$. $\mathrm{PZ}, 0,71 \mathrm{~V}^{62}$ y del sistema Trp, $\mathrm{H}^{+} / \mathrm{Trp}$, $1,02 \mathrm{~V}$.

En general, proteínas y péptidos con residuos Tyr o Trp son los receptores más sensibles en el proceso de transferencia de radicales libres desde hemoproteínas ${ }^{26,51}$, proceso que sería de múltiples consecuencias biológicas. Así mismo, es de destacar que una especie reactiva de la $\mathrm{Mb}$, con un centro activo similar al del cit c activado, interactúa mayormente con Tyr, Trp y Cys libres ${ }^{51}$. Resumiendo, los efectos de los compuestos tiol, Tyr y Trp sugieren que los radicales $\mathrm{FTZ}^{+}$. reaccionan con el grupo $\mathrm{SH}$ de la Cys y los residuos de Tyr y Trp de la LADH, inactivándola. 
La protección de la LADH por NADH frente a radicales $\mathrm{PZ}^{+}$(Tabla 3; Figura 8 ) fue transitoria, y la máxima protección observada hasta $30 \mathrm{~min}$ del inicio de la reacción está en relación con el mayor efecto sobre la concentración del radical. La disminución brusca de la concentración de $\mathrm{PZ}^{+} .(95 \%)$ fue obtenida al añadir NADH cuando el radical catiónico alcanzó el más alto nivel (Tabla 4). El NADH es oxidado rápidamente a NAD por radicales libres o moléculas aceptoras de un electrón con adecuados potenciales de reducción ${ }^{63}$. El potencial redox del sistema NAD, $\mathrm{H}^{+} / \mathrm{NADH}$ es de $0,3 \mathrm{~V}^{64} \mathrm{y}$ el del par.

$\mathrm{PZ}^{+} / \mathrm{PZ}$ es de $0,71 \mathrm{~V}$, luego la siguiente reacción puede ocurrir, con una constante de velocidad, $\mathrm{k}=5 \times 10^{5} \mathrm{M}^{-1} \mathrm{~s}^{-1} 65$ :

$\mathrm{NADH}+\mathrm{PZ}^{+} \rightarrow \mathrm{NAD}+\mathrm{H}^{+}+\mathrm{PZ}(10)$

donde el radical $\mathrm{PZ}^{+}$. oxida al NADH generando NAD y la molécula neutra PZ. El radical NAD reacciona fácilmente con el oxígeno molecular $(k=$ $\left.1,9 \times 10^{9} \mathrm{M}^{-1} \mathrm{~s}^{-1}\right)^{66}$ generando anión superóxido y $\mathrm{NAD}^{+}$. La reacción 10 indica la función antioxidante directa del $\mathrm{NADH}$, de especial importancia a nivel mitocondrial ${ }^{65}$. Sin embargo, NAD y anión superóxido son potencialmente citotóxicos. Recientemente se demostró que el NADH y otros sustratos pueden ser oxidados por el intermediario tipo compuesto I del $\mathrm{cit}^{33}, \mathrm{y}$ aunque en nuestro estudio la velocidad de oxidación del NADH por cit c $/ \mathrm{H}_{2} \mathrm{O}_{2}$ fue solo de $1,2 \mu \mathrm{M} / \mathrm{min}$ (no mostrado), no puede descartarse un efecto del NADH como inhibidor de la peroxidación de FTZ, actuando competitivamente frente al cit c activado. Al reemplazar el cit c por una peroxidasa, el consumo de NADH durante la reacción fue seguido por reanudación de la formación del radical $\mathrm{PZ}^{+.14}$, lo cual explicaría la disminución del efecto protector observado al prolongar el tiempo de incubación (Figura 8). Estas observaciones sugieren un nuevo estudio del efecto del NAD y del anión superóxido sobre la inactivación de la LADH por el sistema cit c/ $\mathrm{H}_{2} \mathrm{O}_{2}$. El ascorbato y el trolox (análogo soluble de la vitamina $\mathrm{E}$ ), fueron los mejores protectores de la $\mathrm{LADH}$ frente al radical $\mathrm{PZ}^{+}$generado en el sistema cit $\mathrm{c} / \mathrm{H}_{2} \mathrm{O}_{2}$ (Tabla 3 ). Este efecto concuerda con la gran capacidad de estos compuestos para desactivar radicales $\mathrm{FTZ}^{+}$. (Tabla 4), formando la FTZ original y el radical ascorbil ${ }^{17,53,62}$, o el radical fenoxilo del trolox ${ }^{14}$.
Estudios previos han sugerido que el ascorbato es sustrato del cit c activado ${ }^{26,33}$, y en las condiciones del presente trabajo hemos demostrado la interacción cit c/peróxido con el ascorbato en ausencia y presencia de PZ (comunicación personal); y siendo el trolox sustrato de peroxidasas ${ }^{67}$, es un posible sustrato del intermediario reactivo del cit c. Entonces, la inhibición de la formación de $\mathrm{PZ}^{+}$. cuando el ascorbato o el trolox estuvieron presentes en el sistema experimental antes de iniciar la reacción con el $\mathrm{H}_{2} \mathrm{O}_{2}$, puede explicarse, en parte, por la participación de estos antioxidantes como sustratos competitivos de las FTZ por el cit c.

Por otra parte, este estudio revela mayor susceptibilidad de la LADH de $T c$ en comparación con la enzima de mamífero, lo cual sustenta la hipótesis de considerar la enzima del parásito como blanco para el diseño de fármacos específicos $^{3}$ estructuralmente relacionados a las FTZ. Estas observaciones realizadas con sistemas cit c son concordantes con los resultados encontrados en otros sistemas generadores de radicales $\mathrm{FTZ}^{+}$, MPO/ $\mathrm{H}_{2} \mathrm{O}_{2}(14)$ y $\mathrm{HRP}(\mathrm{Mb}) /$ $\mathrm{H}_{2} \mathrm{O}_{2}$ (comunicación personal).

El $T c$ es fagocitado por los macrófagos y puede penetrar en otras células, incluyendo células musculares del miocardio y del sistema retículo endotelial ${ }^{10,68,69}$. El tratamiento con CPZ o TFPZ de cardiomiocitos infectados con $T c$ alteró la membrana plasmática y los microtúbulos subpeliculares del parásito intracelular ${ }^{8}$. La PMTZ también desorganiza las membranas celulares de tripomastigotes de $T c^{70}$, y la $\operatorname{TFP}^{9}$ y $\mathrm{TRDZ}^{10}$ generan lesiones mitocondriales del parásito, lo cual puede implicar acciones de las FTZ desde el interior del $T c$ y de las células huésped. La peroxidación de las FTZ, especialmente PZ, TRDZ y TMPZ, podría ocurrir por el cit c de las células huésped y del $T c$ si se alcanzan apropiadas concentraciones "in situ" de $\mathrm{H}_{2} \mathrm{O}_{2}$. La acción del cit c sería a nivel mitocondrial o citoplasmático; en este último compartimiento por el cit c liberado durante la apoptosis que puede ocurrir en el curso de la infección con $T c^{71}$. El cit c del $T c^{72,73}$ y la actividad peroxidasa de las mitocondrias de tripanosomátidos han sido estudiados ${ }^{74}$, y ésta actividad puede atribuírse al cit c del parásito. Es interesante anotar que la oxidación del cit c por cloramina- $\mathrm{T}^{75}$ o por $\mathrm{HOCl}, \mathrm{HOBr}$, y HOI- ${ }^{76}$ metabolitos de los neutrófilos activados- cambia sus propiedades redox, habiéndose observado, 
además, aumento de la actividad peroxidasa del cit c especialmente por la reacción con $\mathrm{HOCl}^{77}$, por iodinación, por nitración con peroxinitrito ${ }^{78}$ y otros factores ${ }^{79,80}$. El aumento de la actividad cit c peroxidasa ocurre por desplazamiento del sexto ligando de coordinación Fe Hemo-Met ${ }^{80}$, con oxidación de ese residuo de Met, disfunción del cit $\mathrm{c}$ en el transporte de electrones y, posiblemente, aumento de la generación de anión superóxido y de $\mathrm{H}_{2} \mathrm{O}_{2}{ }^{77}$. Previamente se había establecido que la interrupción del flujo de electrones a nivel del cit $\mathrm{c}$ en la cadena respiratoria mitocondrial durante la apoptosis, aumenta la producción de radicales superóxido y su dismutación a $\mathrm{H}_{2} \mathrm{O}_{2}$ y $\mathrm{O}_{2}(20)$. El sistema cit c/ $\mathrm{H}_{2} \mathrm{O}_{2} / \mathrm{FTZ}$ puede ser una causa potencial del efecto tripanocida de las FTZ, y aunque los interceptores de radicales $\mathrm{FTZ}^{+}$-GSH, Tyr, Trp, NADH y otros compuestos-, pueden prevenir la inactivación de la LADH $T c$, generan radicales secundarios potencialmente citotóxicos y disminuyen la concentración de estos importantes metabolitos celulares. En conjunto, estos efectos pueden desempeñar un rol destacado en la acción anti-T. cruzi y en otras manifestaciones farmacológicas de las FTZ.

\section{RESUMEN}

El citocromo c catalizó la oxidación de las fenotiazinas (FTZ) en presencia de peróxido de hidrógeno. La formación del radical catiónico de promazina $\left(\mathrm{PZ}^{+}\right)$se demostró por espectrofotometría y por su conversión a promazina sulfóxido La dihidrolipoamida deshidrogenasa (LADH) del Trypanosoma cruzi es inhibida irreversiblemente por el sistema citocromo c/ $\mathrm{H}_{2} \mathrm{O}_{2}$ complementado con fenotiazinas. La inactivación de la LADH del parásito varía según la estructura de las FTZ, el tiempo de incubación del sistema pro-oxidante con la LADH, y la presencia de un antioxidante supresor de radicales $\mathrm{FTZ}^{+}$Entre las $12 \mathrm{FTZ}$ ensayadas, la promazina (PZ), tioridazina (TRDZ) y trimeprazina (TMPZ) fueron las más efectivas produciendo inactivaciones de $82 \%, 76 \%$ y $72 \%$, respectivamente, a los 90 min de incubación. El efecto de PZ (con grupo alquilamino en la posición $\mathrm{N}$ 10) disminuyó por modificación de su estructura en la posición 2 (efecto inactivante de $\mathrm{PZ}>$ cloropromazina $(\mathrm{CPZ})>$ propionilpromazina $(\mathrm{PPZ})>$ trifluopromazina (TFPZ) o en la posición
10 ( efecto inactivante de $\mathrm{PZ}>\mathrm{TMPZ}>$ prometazina (PMTZ).El efecto de las FTZ con sustituyente piperidinil en N 10 dependió del grupo de la posición 2 ( $\mathrm{SCH}_{3}$ en TRDZ de mayor efecto; $\mathrm{CN}$, en propericiazina (PCYZ), la de menor efecto entre las FTZ estudiadas). Parece que la presencia del sustituyente piperazinil en posición $\mathrm{N} 10$ no tiene función importante en el efecto inactivante de las FTZ, el cual dependió de la estructura del grupo en la posición 2. El efecto de los compuestos con $\mathrm{Cl}$ en posición 2 $(\mathrm{CPZ}$, procloroperazina $(\mathrm{PCP})$, perfenazina (PFZ)) fue mayor que el obtenido con los compuestos $\mathrm{CF}_{3}$ (TFPZ, trifluoroperazina (TFP), flufenazina (FFZ), e independiente de la estructura del sustituyente N 10.El efecto de las FTZ sobre la LADH de T. cruzi depende, por lo menos en parte, de la estabilidad de los radicales $\mathrm{FTZ}^{+}$. generados por la actividad peroxidasa. La LADH $T$ c, en comparación con la LADH de mamífero, presentó mayor sensibilidad al efecto inactivante de los radicales catiónicos de varias FTZ estudiadas.Los compuestos tiol (GSH, Cys, NAC, PAM, CPT), Tyr, Trp, NADH, ascorbato y trolox, protegieron a la LADH frente a los radicales $\mathrm{PZ}^{+}$, suprimiendo su reactividad. $\mathrm{Se}$ plantea la posible participación del citocromo c en el mecanismo de la acción tripanocida de las FTZ, mediante la generación de radicales $\mathrm{FTZ}^{+}$. y consiguiente inactivación de la LADH o la acción de los radicales libres secundarios y el consumo de metabolitos como resultado de la actividad de los antioxidantes.

Abreviaturas: LADH, dihidrolipoamida deshidrogenasa; $T$ c,Trypanosoma cruzi; citocromo c, cit c; MPO, mieloperoxidasa; Mb, mioglobina; FTZ, fenotiazina, dibenzotiazina;PZ, promazina, 10 -(3-dimetilaminopropil)FTZ;CPZ,cloropromazina,2-cloro-10-(3dimetilamino propil)-FTZ; PPZ, propionilpromazina, 2-propionil-10-(3-dimetilaminopropil)-FTZ; TFPZ, trifluopromazina,2trifluorometil-10-(3-dimetilamino propil)-FTZ; TMPZ, trimeprazina, 10-(2-metil-3-dimetilaminopropil)-FTZ; PMTZ, prometazina, 10-(2dimetilaminopropil)-FTZ; TRDZ, tioridazina, 2metilmercapto-10-[2-(1-metil-2-piperidinil)-etil]FTZ; PCYZ, propericiazina, 2 ciano-10-[3-(4 hidroxi-1 piperidinil)- propil]-FTZ; PCP, procloroperazina, 2-cloro-10-[3-(1-metil-4piperazinil)-propil]-FTZ; PFZ, perfenazina, 2c loro- $10-[3-[1-(2-\mathrm{hidroxi}$ etil $)-4$ - 
piperazinil]propil]-FTZ; TFP, trifluoperazina, 2trifluorometil-10-[3-(1-metil-4-piperazinil)propil]-FTZ; FFZ, flufenazina, 2- trifluorometil10-[3-[1-(2-hidroxietil)-4-piperazinil] propil]FTZ; NAC, N-acetilcisteína; PAM, penicilamina; CPT captopril;Trolox, 6-hidroxi 2,5,7,8tetrametilcromano-2-ácido carboxílico.

\section{REFERENCIAS}

1.- URBINA J A, DOCAMPO R. Specific chemotherapy of Chagas disease: controversies and advances. Trends in Parasitology 2003; 19: 495-50.

2.- WORLD HEALTH ORGANIZACIÓN Control of Chagas Disease. Tech. Rep Ser 2002; 905: 1-109.

3.- KRAUTH-SIEGEL R L, SCHÖNECK R. Trypanothione reductase and lipoamide dehydrogenase as target for a structure-based drug design. FASEB J 1995; 9: 113846 .

4.- MOTOHASHI N, KAWASE M, MOLNAR J, et al. Antimicrobial Activity of N-Acylphenothiazines and Their Influence on Lipid Model Membranes and Erythrocyte Membranes. Arzneim Forsch Drug Res 2003; 53: 590-9.

5.- MOTOHASHI N, KURIHARA T, KAWASE M, et al. Drug resistance reversal, anti-mutagenicity and antiretroviral effect of phtalimido- and chloroethylphenothiazines. Anticancer Res 1997; 17: 3537-43.

6.- OLIVEIRA M M, ANTUNES A, MELLO F G. Growth of T. cruzi epimastigotes controlled by shifts in cyclic AMP mediated by adrenergic ligands. Mol Biochem Parasitol 1984; 11: 283-92.

7.- TELLEZ-IÑON M T, ULLOA R M, TORRUELA M, TORRES H N. Calmodulin and $\mathrm{Ca}_{2}{ }^{+}$- dependent cAMP phosphodiesterase activity in Trypanosoma cruzi. Mol Biochem Parasitol 1985; 17: 143-53.

8.- DE CASTRO S L,SOEIRO M N C, MEIRELLES MDENL. Trypanosoma cruzi: Effect of phenothiazines on the parasite and its interacción with host cells. Mem Inst Oswaldo Cruz 1992; 87: 209-15.

9.- LACUARA J L, BARIOGLIO S R, PAGLINI-OLIVA $P$, et al. Disruption of mitochondrial function as the basis of the trypanocidal effect of trifluoperazine on Trypanosoma cruzi. Experientia 1991; 47: 612-6.

10.- PAGLINI-OLIVA P, FERNÁNDEZ A R, FRETES R, PESLMAN A. Structural, ultrastructural studies and evolution of Trypanosoma cruzi infected mice treated with thioridazine. Exp.Mol Pathol 1998; 65: 78-86.

11.- RIVAROLA H W, FERNÁNDEZ A R, ENDERS J E, et al. Thioridazine treatment modifies the evolution of Trypanosoma cruzi infection in mice. Ann Trop Med Parasitol 1993: 695-702.

12.- CHAN C, YIN H, GARFORTH J, et al. Phenothiazine inhibitors of trypanothione reductase as potential antitrypanosomal and antileishmanial drugs. J Med Chem 1998; 41: 148-56.

13.- GUTIÉRREZ-CORREA J, FAIRLAMB A H, STOPPANI A O M. Inactivación de la tripanotiona reductasa de Trypanosoma cruzi por radicales libres catiónicos de fenotiazinas. Rev Arg Microbiol 2001; 33: 36-46.
14.- GUTIÉRREZ-CORREA J, KRAUTH-SIEGEL R L, STOPPANI A O M. Phenothiazine Radicals Inactivate Trypanosoma cruzi Dihydrolipoamide Dehydrogenase: Enzyme Protection by Scavengers. Free Rad Res 2003; 23: 281-91.

15.- WILLIAMS C H J. Lipoamide deshidrogenase, glutathione reductase, thioredoxin reductase and mercuric reductase. In: Müller F. Ed. A Family of Flavoenzyme Transhydrogenases in Chemistry and Biochemistry of Flavoenzymes (CRC Press. Boca Raton, FL) 1992; 3: 121-211.

16.- DOCAMPO R, BOISO J F DE, STOPPANI A O M. Tricarboxylic acid cycle operation at the kinetoplast mitochondrion complex of Trypanosoma cruzi. Biochim Biophys Acta 1978; 502: 466-76.

17.- SCHÖNECK R, BILLAUT-MULOT O, NUMRICH P, et al. Cloning, sequencing and functional expression of dihydrolipoamide dehydrogenase from human pathogen Trypanosoma cruzi. Eur J Biochem 1997; 243: 73947.

18.- BANDO M, OBAZAWA H, TANIKAWA T. Scavenging of chlorpromazine cation radical by ascorbic acid or glutathione. Free Rad Biol Med 1986; 2: 261-6.

19.- BATTISTUZZI G, BORSARI M, SOLA M. Redox Properties of Cyochrome c Antioxid, Redox Signal 2001; 3: 279-91.

20.- DICKERSON R E, TIMKOVICH R. En. The Enzymes (Boyer P, ed) 11, 3rd Ed, Academic Press, New York. 1975; 397-547.

21.- CAI J, JONES D P. Superoxide in Apoptosis. Mitochondrial generation triggered by cytochrome $\mathrm{c}$ loss. J Biol Chem 1998; 273: 11401-4.

22.- CAI J, YANG J, JONES D P. Mitochondrial control of apoptosis: the role of cytochrome c. Biochim Biophys Acta 1998; 1366: 139-49.

23.- BARR D P, GUNTHER M R, DETERDING L J, et al. ESR Spin-trapping of a Protein-derived Tyrosyl Radical from. the Reaction of Cytochrome c with Hydrogen Peroxide. J Biol Chem 1996; 271: 15498-503.

24.- CADENAS E, BOVERIS A, CHANCE B. Chemiluminescence of lipid vesicles supplemented with cytochrome c and hydroperoxide. Biochem J 1980; 188: $577-83$.

25.- CHEN Y R, CHEN C L, CHEN W, et al. Formation of proteín tyrosine ortho-semiquinone radical and nitrotyrosine from cytochrome c derived tyrosyl radical. J Biol Chem 2004; 279: 18054-62.

26.- DETERDING L J,BARR D P, MASON R P,TOMER K R. Characterization of Cytochrome $c$ Free Radical Reactions with Peptides by Mass Spectrometry. J Biol Chem 1998; 273: 12863-9.

27.- RADI R, SIMS S, CASSINA A, TURRENS J F. Roles of Catalase and Cytochrome $\mathrm{C}$ in HydroperoxideDependent Lipid Peroxidation and Chemiluminescence in Rat Heart and Kidney Mitochondria. Free Rad Biol Med 1993; 15: 653-9.

28.- HAREL S, KANNER J The generación of Ferryl or Hydroxyl Radicals during interaction of Haemproteins with Hydrogen Peroxide. Free Rad Res Comms 1988; 5: 21-33.

29.- HAYAKAWA M, OGAWA T, SUGIYAMA S, OZAWA T. Hydroxyl radical and Leukotoxin 
Biosynthesis in Neutrophil Plasma Membrane. Biochem Biophys Res Commun 1989; 161: 1077- 85.

30.- RADI R, THOMSON L, RUBBO H, PRODANOV E. Cytochrome c-Catalyzed Oxidation of Organic Molecules by Hydrogen Peroxide. Arch Biochem Biophys 1991; 288: 112-7.

31.- AKASAKA R,MASHINOT, HIROBE M. Cytochrome P450-like Substrate Oxidation Catalyzed by Cytochrome c and Immobilized Cytochrome c. Arch Biochem Biophys 1993; 301: 355-60.

32.- BURKITT M J, WARDMAN P. Cytochrome C is a potent catalyst of dichlorofluorescin oxidation: implications for the role of reactive oxygen species in apoptosis. Biochem Biophys Res Commun 2001; 282: 329-33.

33.- LAWRENCE A, JONES CM, WARDMAN P, BURKITT M J. Evidence for the role of a peroxidase compound I-type intermediate in the oxidation of glutathione. NADH, ascorbate, and dichlorofluorescin by cytochrome $\mathrm{c} / \mathrm{H}_{2} \mathrm{O}_{2}$. Implications for oxidative stress during apoptosis. J Biol Chem 2003; 278: 29410 9.

34.- VAZQUEZ-DUHALT R. Cytochrome c as a biocatalyst. J Mol Catalysis B: Enzymatic 1999; 7: 241-9.

35.- ROSEI M A, BLARZINO C, COCCIA R, et al. Production of melanin pigments by cytochrome c/ $\mathrm{H}_{2} \mathrm{O}_{2}$ System. Int J Biochem Cell Biol 1998; 30: 457419 .

36.- FORMAN H J, AZZI A. On the virtual existence of superoxide anions in mitochondria: thoughts regarding its role in pathophysiology. FASEB J. 1997; 1: 3744375.

37.- CHANCE B, SIES H, BOVERIS A. Hydroperoxide in mammalian organs. Physiol Rev 1979; 59: 527-605.

38.- FLAHERTY J T, WEISFELDT M Reperfusion Injury. Free Rad Biol Med 1988; 5: 409-419.

39.- KELDER P P, FISCHER M J E, DE MOL N J, JANSSEN L H M. Oxidation of chlorpromazine by methemoglobin in the presence of hydrogen peroxide. Formation of chlorpromazine radical cation and its covalente binding to methemoglobin. Arch Biochem Biophys 1991; 284: 313-9.

40.- KELDER P P, DE MOL N J, FISCHER M JE, JANSSEN L H M. Kinetic evaluation of the oxidation of phenothiazine derivatives by methemoglobin and horseradish peroxidase in the presence of hydrogen peroxide. Implications for the reaction mechanisms. Biochim Biophys Acta 1994; 1205: 230-8.

41.- GALZIGNA L, RIZZOLI V, SCHIAPPELLI MP, et al. Horseradish peroxidase-catalyzed sulfoxidation of promethazine and properties of promethazine sulfoxide. Free Rad Biol Med 1996; 20: 807-11.

42.- DESSER R K, HIMMELHOCH S R, EVANS W H, et al. Guinea pig heterophil and eosinophil peroxidase. Arch Biochem Biophys 1972; 148: 452-65.

43.- PIETTE L H, BULOW G, YAMAZAKI Y. Electron paramagnetic resonance studies of the chlorpromazine free radical formed during enzymic oxidation by peroxidase-hydrogen peroxide. Biochim Biophys Acta 1964; 88: 120-9.

44.- VAZQUEZ A, TUDELA J, VARÓN R, GARCÍACÁNOVAS F. Determination of the molar absorptivities of phenothiazine cation radicals generated by oxidation with hydrogen peroxide/peroxidase. Anal Biochem 1992; 202: 245-8.

45.- LEVY L,TOZER TN, LOVELAND T B. Stability of some phenothiazine free radicals. J Med Chem 1991; 15: 898-905.

46.- BANCI L,BERTTINI I, LIU G, et al. Effects of extrinsic imidazole ligation on the molecular and electronic structure of cytochrome c. J Biol Inorg Chem 2001; 6: 628-37.

47.- DIEDERIX R E M,UBBINK M, CANTERS G W. The peroxidase activity of cytochrome c-550 from. Paracoccus versutus. Eur J Biochem 2001; 268: 4207 16.

48.- PAN D, SHOUTE LCT, PHILliPS D L.Time Resolved Resonance Raman and Density Functional Study of the Radical Cation of Chlorpromazine. J Phys Chem A 2000; 104: 4140-8.

49.- GIULIVI C, CADENAS E. Heme protein radicals: formation, fate, and biological consequences. Free Radic Biol Med 1998; 15: 269-79.

50.- GUNTHER M R, STURGEON B S, MASON RP. A long-lived tyrosyl radical from the reaction between horse methmyoglobin and hydrogen peroxide. Free Radic Biol Med 2000; 28: 709-19.

51.- IRWIN J A, OSTDAL H, DAVIES MJ. Myoglobin-induced oxidative damage: Evidence for radical transfer from oxidized myoglobin to other proteins and antioxidants. Arch Biochem Biophys 1999; 362: 94-104.

52.- HORI H, FENNA RE, KIMURA S, IKEDA-SAITO M. Aromatic substrate molecules bind at the distal heme pocket of myeloperoxidase. J Biol Chem 1994; 169 : 8388-92.

53.- FORNI L G, MÖNIG J, MORA-ARELLANO V O, WILLSON R L.Thiyl Free Radicals: Direct Observations of Electron Transfer Reactions with Phenothiazines and Ascorbate. J Chem Soc Perkin Trans 1983; 2: 961-5.

54.- MARQUEZ L A, DUNFORD H B. Kinetics of oxidation of tyrosine and dityrosine by myeloperoxidase compounds I and II. J Biol Chem 1995; 270: 30434-40.

55.- TIEN M. Myeloperoxidase-catalyzed oxidation of tyrosine. Arch Biochem Biophys 1999; 367: 61-6.

56.- GOODWIN D C, GROVER T A, AUST S D. Redox Mediation in the Peroxidase-Catalyzed Oxidation of Aminopyrine: Possible Implications for Drug-Drug Interactions. Chem Res Toxicol 1996; 9: 476-83.

57.- GOODWIN D C, GROVER T A, AUST S D. Roles of Efficient Substrates in Enhancement of PeroxidaseCatalyzed Oxidations. Biochemistry 1997; 36: 13947.

58.- OLORUNNIJI F J, MALOMO S O, ADEDIRAN S A, ODUTUGA A A. Promethazine Oxidation by Redox Mediation in Peroxidase Reactions. Arch Biochem Biophys 2000; 380: 251-6.

59.- KAPIOTIS S, HERMANN M, HELD I, et al. Tyrosine: an inhibitor of LDL oxidation and endothelial cell cytotoxicity initiated by superoxide/nitric oxide radicals. FEBS Lett 1997; 409: 323-6.

60.- MOOSMANN B, BEHL C. Cytoprotective antioxidant function of tyrosine and tryptophan residues in transmembrane proteins. Eur J Biochem 2000; 267: 5687-92. 
61.- MOOSMANN B, BEHL C. Secretory peptide hormones are biochemical Antioxidants:Structure-Activity Relationship. Mol Pharmacol 2002; 61: 260-8.

62.- PELIZZETTI E, MEISEL D, MULAC W A, NETA P. On the Electron Transfer from Ascorbic Acid to various Phenothiazine Radicals. J Am Chem Soc 1979; 101: 6954-9.

63.- SCHELLENBERG K A, HELLERMAN L. Oxidation of reduced diphosphopyridine nucleotide. J Biol Chem 1958; 231: 547-56.

64.- FARRINGTON J A, LAND E J, SWALLOW J. The one-electron reduction potentials of NAD. Biochim Biophys Acta 1980; 590: 273-6.

65.- KIRSCH M, DE GROOT H. NAD (P) H, a directly operating antioxidant? FASEB J. 2001; 15: 1569-74.

66.- LAND E J, SWALLOW A J. One-electron reactions in biochemical systems as studied by pulse radiolysis. IV. Oxidation of dihydronicotinamide-adenine dinucleotide. Biochim Biophys Acta 1971; 234: $34-$ 42.

67.- NAKAMURA M, HAYASHI T. Oxidation Mechanism of Vitamin E Analogue (Trolox C, 6 hydroxy2,2,5,7,8-pentamethylchroman) and Vitamin E by Horseradish Peroxidase and Myoglobin. Arch Biochem Biophys 1992; 299: 313-9.

68.- RIMOLDI M T, OLABUENAGE S E, ELIZALDE DE BRACCO M M. Phagocytosis of Trypanosoma cruzi by human polymorphonuclear leukocytes. J Protozool 1981; 28: 351-4.

69.- STEENKAMP D J. Trypanosomal Antioxidants and Emerging Aspects of Redox Regulation in the Trypanosomatids. Antioxid, Redox Signal 2002; 4: 105-21.

70.- FERNÁNDEZ A R, FRETES R, RUBIALES S, et al. Trypanocidal effects of promethazine. Medicina (Buenos Aires) 57: 59-63.

71.- LOPES M F, DOS REIS G A. Experimental Chagas disease: Phagocytosis of apoptotic lymphocytes deactivates macrophages and fuels parasite growth. Apoptosis 2000; 5: 221-4.

72.- SCHWARCZDE TARLOVSKY MN, AFFRANCHINO J L,STOPPANI A O M, et al. Citocromos en diferentes estadios, cepas y poblaciones de Trypanosoma cruzi.
Rev Arg Microbiol 1985; 17: 121-30.

73.- HILL G C, GUTTERIDGE W E, MATHEWSON N W. Purification and properties of cytochromes c from. Trypanosomatids. Biochim Biophys Acta 1971; 243: 225-9.

74.- KUSEL J P, BOVERIS A, STOREY B T. $\mathrm{H}_{2} \mathrm{O}_{2}$ Production and Cytochrome c Peroxidase Activity in Mitochondria Isolated from. the Trypanosomatid Hemoflagellate Crithidia fasciculata. Arch Biochem Biophys 1973; 158: 799-805.

75.- PANDE J, KINNALLY K, THALUM K K, et al. Methionine oxidized horse heart cytochromes c.I Reaction with chloramine- T, products, and their oxidoreduction properties. J Protein Chem 1987; 6: 295-319.

76.- PRUTZ W A, KISSNER R, NAUSER T, KOPPENOL W H. On the oxidation of cytochrome $\mathrm{c}$ by hypohalous acids. Arch Biochem Biophys 2001; 389: 110-22.

77.- CHEN Y-R, DETERDING L J, STURGEON B E, et al. Protein Oxidation of Cytochrome c by Reactive Halogen Species Enhances Its Peroxidase Activity. J Biol Chem 2002; 277: 29781-91.

78.- CASSINA A M, HODARA R, SOUZA J M, et al. Cytochrome c Nitration by Peroxynitrite. J Biol Chem 2000; 275: 21409-15.

79.- DIEDERIX R E M,UBBINK M, CANTERS G W. Peroxidase Activity as a Tool for Studying the Folding of c-Type Cytochromes. Biochemistry 2002; 41: 13067-77.

80.- NANTES I L, ZUCCHI M R, NASCIMENTO O R, FALJONI-ALARIO A. Effect of Heme Iron Valence State on the Conformation of Cytochrome $\mathrm{c}$ and Its Association with Membrane Interfaces. A CD and EPR Investigation. J Biol Chem 2001; 276: 153-8.

Agradecimientos: Este trabajo se realizó con apoyo de la Universidad de Buenos Aires y de su Hospital de Clínicas José de San Martín; de la Universidad Nacional Mayor de San Marcos y de su Instituto de Medicina Tropical Daniel A. Carrión; de la Prof..Dr. R.L. Krauth- Siegel (BiochemieZentrum, Heidelberg University, Germany), quien contribuyó decisivamente con la enzima LADH T. cruzi; y, de los Laboratorios Roemmers. 